\title{
Numerical analysis for an energy-stable total discretization of a poromechanics model with inf-sup stability
}

\author{
B. Burtschell ${ }^{1,2}$, P. Moireau ${ }^{1,2}$, D. Chapelle ${ }^{1,2 *}$ \\ ${ }^{1}$ Inria, Université Paris-Saclay \\ ${ }^{2}$ LMS, Ecole Polytechnique, CNRS
}

\begin{abstract}
We consider a previously proposed general nonlinear poromechanical formulation, and we derive a linearized version of this model. For this linearized model, we obtain an existence result and we propose a complete discretization strategy - in time and space - with a special concern for issues associated with incompressible or nearly-incompressible behavior. We provide a detailed mathematical analysis of this strategy, the main result being an error estimate uniform with respect to the compressibility parameter. We then illustrate our approach with detailed simulation results and we numerically investigate the importance of the assumptions made in the analysis, including the fulfillment of specific inf-sup conditions.
\end{abstract}

\section{Introduction}

Poromechanical models endeavor to represent the mechanical behavior of a multi-phase continuum within the framework of the so-called mixture theory, by which each phase is assumed to occupy the whole geometric domain, while the volume ratio of all phases can vary in time and space [10]. In [6] a general two-phase - with a solid and an incompressible fluid - poromechanical model was specifically formulated to be able to represent large strains and significant fluid inertia, based on fundamental physical principles. A typical application motivating this model with such challenging features was provided by the perfusion of blood within the muscles, but other examples abound, in life sciences in particular, e.g. with the mechanics of spongy tissues, lungs, or even cells. The resulting equations were found to bear some interesting close similarity to fluid-structure interaction systems written in an Arbitrary Lagrangian-Eulerian formalism, albeit with here the deformation of the geometry given by that of the solid domain - hence, without any arbitrary character - and with additional volume-distributed interaction forces.

Regarding the discretization of such equations, the issue of time discretizations devised to be consistent with energy balances is known to be crucial already in the realm of classical fluidstructure interaction systems $[11,12,14]$. The formulation of adequate energy-preserving time discretization schemes for the model of [6] was addressed in this same paper with a monolithic time scheme, and later specifically treated in [5] with the objective of proposing a partitioned time scheme, a more tractable approach in numerical practice. By contrast, the question of spatial discretization for this specific poromechanical model has not yet been raised. This is not a serious shortcoming in most cases, as in particular the fluid equations in the coupled system are not associated with an incompressibility constraint - in the sense of local volume preservation - even though the fluid itself is actually incompressible, which is explained by the fact that volume may vary due to solid material compressibility. However, in some applications it may

\footnotetext{
${ }^{*}$ Corresponding author: dominique.chapelle@inria.fr
} 
be required to consider a quasi-incompressible solid, in which case a new constraint equation - more complex than standard fluid incompressibility - arises in the (solid) incompressible limit, and this motivates the present work.

The objective in this paper is to devise a total discretization - in both time and space - of a poromechanical model of the above type, with a complete numerical analysis in order to establish that the error estimate is robust with respect to the incompressible limit on the solid side. As regards time discretization, a mid-point scheme is considered for the solid equations, while pressure and fluid velocity are handled with Euler-backward, and a discrete energy estimate is proven to hold, including with an additional ingredient of Robin-type boundary condition "à la Nitsche" to penalize fluid-structure kinematical coupling at interfaces, as proposed and analysed for fluid-structure interaction problems in $[4,11]$. For spatial discretization, we consider a global finite element approach in the coupled mixed system, and this involves some non-standard infsup stability issues. The numerical analysis itself is performed with a linearized version of the poromechanical model, but we also provide numerical illustrations of the same discretization procedures used with the original nonlinear model, which substantiates the relevance of the approach.

Concerning the positioning of this work, the necessity of exercising special care to handle the discretization of incompressible fluid formulation has been a subject of intensive studies for decades, with mathematically rigorous and practically effective solutions obtained with mixed formulations, in particular - see [2] and references therein - and with natural extensions in the realm of porous flows within rigid solids. The numerical questions raised when considering deformable solids - i.e. poromechanics - have been studied more recently, see in particular $[9,17]$ who proposed finite element spaces and conducted numerical studies to investigate the impact of nearly-incompressible solid behavior. Our main contribution here lies in providing a complete mathematical analysis of a proposed total discretization procedure, with detailed error estimates obtained under specific assumptions, including special inf-sup conditions.

The outline of the paper is as follows. In Section 2 we describe the problem setting, i.e. we recall the equations of the general (nonlinear) poromechanical model of [6], we derive a linearized version of this model for which existence is established, and we propose a complete discretization strategy for this linearized formulation. Then Section 3 is devoted to the mathematical analysis of the discretization, and the main result is an error estimate uniform with respect to solid compressibility. Next, Section 4 provides numerical illustrations of our approach, and also a numerical assessment of the importance of the discrete inf-sup conditions under which the error estimate was obtained. Finally, we give some concluding remarks in Section 5.

\section{Problem setting}

We will start in Section 2.1 by summarizing the general - non-linear - poromechanical model previously proposed in [6], before proceeding to linearize the equations around the solution at rest (Section 2.2), and then analysing the mathematical properties of the resulting linear equations (Section 2.3).

\subsection{Initial nonlinear poromechanical formulation}

Following [6], we consider a general poromechanical model made of a two-phase mixture in which a fluid phase and a solid phase are assumed to coexist and interact at each point. We denote by $\phi$ the volume fraction of the fluid phase.

On the one hand, the solid phase is described by a Lagrangian formulation in the reference domain $\Omega^{0}$. Its mass per unit volume in this configuration is denoted by $\rho_{\mathrm{s} 0}$. The deformed 
domain is denoted by $\Omega^{t}$ and is the image of $\Omega^{0}$ by the mapping

$$
\Omega^{0} \ni \underline{\xi} \mapsto \underline{\mathrm{x}}=\underline{\xi}+\underline{u}_{\mathrm{s}}(\underline{\xi}, t) \in \Omega^{t},
$$

where $\underline{u}_{\mathrm{S}}(\underline{\xi}, t)$ is the displacement field defined at every point $\underline{\xi}$ in the reference domain $\Omega^{0}$. The corresponding velocity field is thus given by

$$
\underline{v}_{\mathrm{s}}=\frac{\mathrm{d} \underline{u}_{\mathrm{s}}}{\mathrm{dt}}=\partial_{t} \underline{u}_{\mathrm{s}}(\underline{\xi}, t),
$$

and the associated deformation gradient tensor is

$$
\underline{\underline{F}}=\underline{\underline{\mathbb{1}}}+\underline{\underline{\nabla}}_{\underline{\xi}} \underline{u_{\mathrm{s}}}
$$

with determinant $J=\operatorname{det} \underline{F}$. We emphasize that $J$ represents the local change of volume of the global mixture, to be distinguished from the change of volume of the solid phase itself given by $J(1-\phi) /\left(1-\phi_{0}\right)$, with $\phi_{0}$ the fluid volume fraction in the undeformed configuration. In the sequel, we denote $J_{\mathrm{s}}=J(1-\phi)$. The right Cauchy-Green deformation tensor is given by $\underline{\underline{C}}=\underline{\underline{F}}^{T} \cdot \underline{\underline{F}}$ and the Green-Lagrange strain tensor by $\underline{\underline{e}}=\frac{1}{2}(\underline{\underline{C}}-\underline{\underline{\mathbb{1}}})$.

On the other hand, we consider an internal fluid flow represented by its velocity $\underline{v}_{\mathrm{f}}$ and pressure $p$ defined in the deformed domain $\Omega^{t}$. The fluid is assumed to be incompressible, hence, the fluid mass per unit volume $\rho_{\mathrm{f}}$ is constant. We also introduce the added fluid mass per unit volume $m$ defined in the reference configuration, viz.

$$
m=\rho_{\mathrm{f}}\left(J \phi-\phi_{0}\right) .
$$

The fluid is assumed to be Newtonian, meaning that the fluid Cauchy stress tensor $\underline{\sigma}_{\mathrm{f}}$ in deformed configuration is given by

$$
\underline{\underline{\sigma}}_{\mathrm{f}}=\underline{\underline{\sigma}}_{\mathrm{vis}}\left(\underline{v}_{\mathrm{f}}\right)-p \underline{\underline{\mathbb{1}}} \text {, with } \underline{\sigma}_{\mathrm{vis}}\left(\underline{v}_{\mathrm{f}}\right)=2 \mu_{\mathrm{f}} \underline{\underline{\varepsilon}}\left(\underline{v}_{\mathrm{f}}\right)
$$

We recall that, in the reference configuration, the Cauchy stress tensor gives the second Piola-Kirchhoff stress tensor with the classical transformation rule

$$
\underline{\underline{\underline{ }}}=J \underline{\underline{F}}^{-1} \cdot \underline{\underline{\sigma}} \cdot \underline{\underline{F}}^{-T},
$$

here written for the global stress tensors of the mixture. Therefore, $\underline{\underline{\Sigma}}_{s}$ the contribution of the solid in the second Piola-Kirchhoff stress tensor $\underline{\underline{\Sigma}}$ is given by

$$
\underline{\underline{\Sigma}}_{s}=\underline{\underline{\Sigma}}-\phi J \underline{\underline{F}}^{-1} \cdot \underline{\underline{\sigma}}_{\mathrm{f}} \cdot \underline{\underline{F}}^{-T}=\underline{\underline{\Sigma}}-\phi \underline{\underline{\Sigma}}_{\mathrm{vis}}+\phi p J \underline{\underline{C}}^{-1},
$$

with $\underline{\underline{\underline{\Sigma}}}_{\mathrm{vis}}=J \underline{\underline{F}}^{-1} \cdot \underline{\underline{\sigma}}_{\mathrm{vis}} \cdot \underline{\underline{F}}^{-T}$.

As in [6], we consider a total stress tensor given by

$$
\underline{\underline{\Sigma}}=\phi \underline{\underline{\Sigma}}_{\mathrm{vis}}+\frac{\partial \Psi(\underline{\underline{e}}, m)}{\partial \underline{\underline{e}}}+\frac{\partial \Psi_{v}(\underline{\underline{\underline{e}}})}{\partial \underline{\underline{\underline{e}}}},
$$

with $\Psi(\underline{\underline{e}}, m)$ the Helmholtz free energy of the mixture and $\Psi_{v}(\underline{\underline{e}})$ a viscous pseudo-potential, both of which need to be specified to characterize the constitutive behavior, see Section 2.2 for the example considered in the linearized formulation. Due to fluid incompressibility, $\Psi(\underline{\underline{e}}, m)=$ $\Psi_{\mathrm{s}}\left(\underline{\underline{e}}, J_{\mathrm{s}}\right)-p_{0} J \phi$ with $\Psi_{\mathrm{s}}$ the solid free energy and $p_{0}$ a reference pressure for the fluid, hence, we also have $[6]$

$$
\underline{\underline{\Sigma}}=\phi \underline{\underline{\Sigma}}_{\mathrm{vis}}+\frac{\partial \Psi_{\mathrm{s}}\left(\underline{\underline{e}}, J_{\mathrm{s}}\right)}{\partial \underline{\underline{e}}}+\frac{\partial \Psi_{v}(\underline{\underline{\dot{e}}})}{\partial \underline{\underline{\underline{e}}}}-p J \underline{\underline{C}}^{-1} .
$$


Recalling (1), this gives for the solid contribution

$$
\underline{\underline{\Sigma}}_{\mathrm{S}}=\frac{\partial \Psi(\underline{\underline{e}}, m)}{\partial \underline{\underline{e}}}+\frac{\partial \Psi_{v}(\underline{\underline{\dot{e}}})}{\partial \underline{\underline{e}}}+\phi p J \underline{\underline{C}}^{-1}=\frac{\partial \Psi_{\mathrm{s}}\left(\underline{\underline{e}}, J_{\mathrm{s}}\right)}{\partial \underline{\underline{e}}}+\frac{\partial \Psi_{v}(\underline{\underline{e}})}{\partial \underline{\underline{e}}}-(1-\phi) p J \underline{\underline{C}}^{-1} .
$$

In addition, we have

$$
p=-\frac{\partial \Psi_{\mathrm{s}}\left(\underline{\underline{e}}, J_{\mathrm{s}}\right)}{\partial J_{\mathrm{s}}} .
$$

The general form of the two-phase mixture dynamics in large deformation is given in strong form in [6] as a coupled system, combining in essence the dynamics of the solid deformation, the dynamics of the fluid flow, and the fluid mass conservation equation. It reads

$$
\left\{\begin{array}{cc}
\rho_{\mathrm{s} 0}\left(1-\phi_{0}\right) \frac{\mathrm{d} \underline{v}_{\mathrm{s}}}{\mathrm{dt}}-\underline{\nabla}_{\underline{\xi}} \cdot\left(\underline{\underline{F}} \cdot \underline{\underline{\Sigma}}_{\mathrm{s}}\right)+p J \underline{\underline{F}}^{-T} \cdot \underline{\nabla}_{\underline{\xi}} \phi & \\
-J \phi^{2} \underline{\underline{k}}_{\mathrm{f}}^{-1} \cdot\left(\underline{v}_{\mathrm{f}}-\underline{v}_{\mathrm{s}}\right)=\rho_{\mathrm{s} 0}\left(1-\phi_{0}\right) \underline{f}, & \text { in } \Omega^{0}, \\
\frac{1}{J} \frac{\mathrm{d}}{\mathrm{dt}}\left(\rho_{\mathrm{f}} J \phi \underline{v}_{\mathrm{f}}\right)+\underline{\nabla}_{\underline{\mathrm{x}}} \cdot\left(\rho_{\mathrm{f}} \phi \underline{v}_{\mathrm{f}} \otimes \rho_{\mathrm{f}}\left(\underline{v}_{\mathrm{f}}-\underline{v}_{\mathrm{s}}\right)\right)-\theta \underline{v}_{\mathrm{f}} & \\
+\phi^{2} \underline{k}_{\mathrm{f}}^{-1} \cdot\left(\underline{v}_{\mathrm{f}}-\underline{v}_{\mathrm{s}}\right)-\underline{\nabla}_{\underline{\mathrm{x}}} \cdot\left(\phi \underline{\sigma}_{\mathrm{vis}}\right)+\phi \underline{\nabla}_{\underline{\mathrm{x}}} p=\rho_{\mathrm{f}} \phi \underline{f}, & \text { in } \Omega^{t}, \\
\frac{1}{J} \frac{\mathrm{d}}{\mathrm{dt}}\left(J \rho_{\mathrm{f}} \phi\right)+\underline{\nabla}_{\underline{\mathrm{x}}} \cdot\left(\rho_{\mathrm{f}} \phi\left(\underline{v}_{\mathrm{f}}-\underline{v}_{\mathrm{s}}\right)\right)=\theta, & \text { in } \Omega^{t}
\end{array}\right.
$$

where we employ the same notation for fields defined over the domains $\Omega^{0}$ and $\Omega^{t}-$ e.g., for the velocity fields - which means that composition by the deformation mapping or its inverse is implicitly used. In (4), the so-called permeability tensor $\underline{\underline{k}}_{\mathrm{f}}$ governs the friction forces between the solid and fluid phases, $f$ is the applied distributed force per unit mass, and $\theta$ the fluid mass input per unit volume in the deformed configuration. Note also that the notation $\frac{\mathrm{d}}{\mathrm{dt}}$ always represents the Lagrangian time derivative at $\xi$ fixed, so that for the equations (4b) and (4c) posed in the deformed domain $\Omega^{t}$, this should be understood as

$$
\frac{\mathrm{d} w}{\mathrm{dt}}=\frac{\partial w}{\partial t}+\underline{\nabla}_{\underline{\mathrm{x}}} w \cdot \underline{v}_{\mathrm{s}},
$$

where the partial derivative $\frac{\partial w}{\partial t}$ is here considered at $\underline{x}$ fixed.

The dynamics (4) must be complemented by adequate boundary conditions. Introducing the total traction $\underline{t}$ on the boundary of the domain $\Omega^{t}$, and $\underline{t}_{0}=J\left\|\underline{\underline{F}}^{-T} \cdot \underline{n}_{0}\right\| \underline{t}$ the transported counterpart on the boundary of the reference domain $\Omega^{0}$, we consider the following boundary conditions on complementary parts of the domain boundary:

- On $\Gamma_{D}^{0}$ (or $\Gamma_{D}^{t}$ in the deformed configuration), Dirichlet boundary conditions for both phases

$$
\underline{u}_{\mathrm{s}}=\underline{u}_{\mathrm{s}}^{\mathrm{pr}}, \quad \underline{v}_{\mathrm{f}}=\underline{v}_{\mathrm{f}}^{\mathrm{pr}} ;
$$

- On $\Gamma_{N}^{0}\left(\right.$ or $\left.\Gamma_{N}^{t}\right)$, Neumann boundary conditions - namely, prescribed forces - for both phases together, with proportional repartition of boundary traction

$$
\underline{\underline{\sigma}} \cdot \underline{n}=\underline{t} \Leftrightarrow \underline{\underline{F}} \cdot \underline{\underline{\Sigma}} \cdot \underline{n}_{0}=\underline{t}_{0}, \quad \underline{\underline{\sigma}} \cdot \underline{n}=\underline{t}
$$

- On $\Gamma_{N \text { nof }}^{0}$ (or $\Gamma_{N \text { nof }}^{t}$ ), Neumann boundary condition for the global mixture, but vanishing fluid flux and proportional repartition of tangential boundary traction

$$
\underline{\underline{\sigma}} \cdot \underline{n}=\underline{t} \Leftrightarrow \underline{\underline{F}} \cdot \underline{\underline{\Sigma}} \cdot \underline{n}_{0}=\underline{t}_{0}, \quad \pi_{\tau}\left(\underline{\underline{\sigma}}_{\mathrm{f}} \cdot \underline{n}\right)=\pi_{\tau}(\underline{t}), \quad\left(\underline{v}_{\mathrm{f}}-\underline{v}_{\mathrm{S}}\right) \cdot \underline{n}=0,
$$

where $\pi_{\tau}=\underline{\mathbb{1}}-\underline{n} \otimes \underline{n}$ denotes the projection onto the tangential plane; 
- On $\Gamma_{N \text { nos }}^{0}$ (or $\Gamma_{N \text { nos }}^{t}$ ), Neumann boundary condition for the global mixture with fluid velocity coinciding with the solid velocity (no sliding)

$$
\underline{\underline{\sigma}} \cdot \underline{n}=\underline{t} \Leftrightarrow \underline{\underline{F}} \cdot \underline{\underline{\Sigma}} \cdot \underline{n}_{0}=\underline{t}_{0}, \quad \underline{v}_{\mathrm{f}}=\underline{v}_{\mathrm{s}}
$$

As already justified in [6], the corresponding weak form of (4) with the associated boundary conditions given above is thus given by

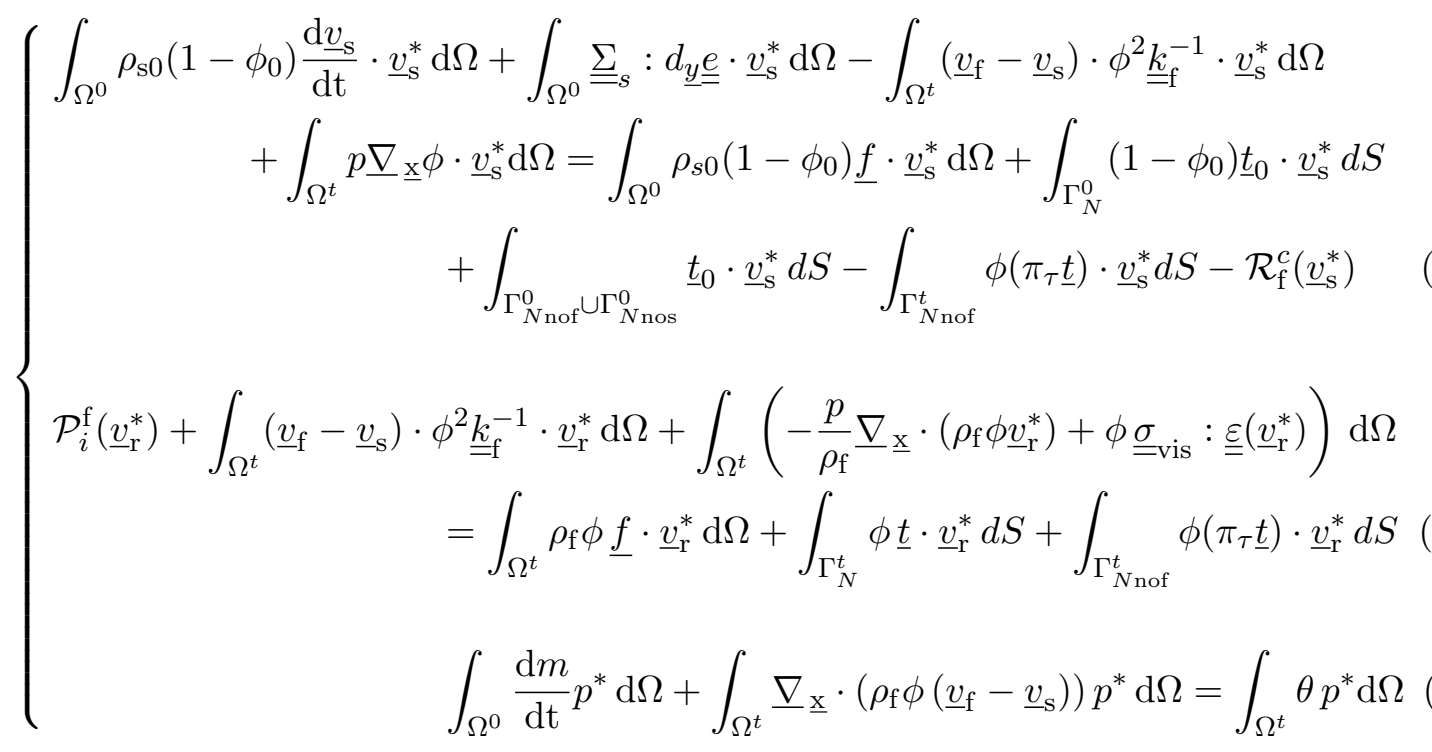

where $\left(\underline{v}_{\mathrm{s}}^{*}, \underline{v}_{\mathrm{r}}^{*}, p^{*}\right)$ denote tests functions and

$$
\mathcal{P}_{i}^{\mathrm{f}}\left(\underline{v}^{*}\right)=\int_{\Omega^{0}} \frac{\mathrm{d}}{\mathrm{dt}}\left(J \rho_{\mathrm{f}} \phi \underline{v}_{\mathrm{f}}\right) \cdot \underline{v}^{*} \mathrm{~d} \Omega+\int_{\Omega^{t}} \underline{\nabla}_{\underline{\mathrm{x}}} \cdot\left(\rho_{\mathrm{f}} \phi \underline{v}_{\mathrm{f}} \otimes\left(\underline{v}_{\mathrm{f}}-\underline{v}_{\mathrm{s}}\right)\right) \cdot \underline{v}^{*} \mathrm{~d} \Omega-\int_{\Omega^{t}} \underline{v}_{\mathrm{f}} \cdot \underline{v}^{*} \mathrm{~d} \Omega,
$$

whereas the continuous residual term $\mathcal{R}_{\mathrm{f}}^{c}$ - associated with fluid reaction forces on boundaries $\Gamma_{N \text { nof }}^{t}$ and $\Gamma_{N \text { nos }}^{t}-$ is given by

$$
\begin{array}{r}
\mathcal{R}_{\mathrm{f}}^{c}\left(\underline{v}_{\mathrm{s}}^{*}\right)=\mathcal{P}_{i}^{\mathrm{f}}\left(\underline{v}_{\mathrm{s}}^{*}\right)+\int_{\Omega^{t}}\left(\underline{v}_{\mathrm{f}}-\underline{v}_{\mathrm{s}}\right) \cdot \phi^{2} \underline{\underline{k}}_{\mathrm{f}}^{-1} \cdot \underline{v}_{\mathrm{s}}^{*} \mathrm{~d} \Omega+\int_{\Omega^{t}}\left(-\frac{p}{\rho_{\mathrm{f}}} \underline{\nabla}_{\mathrm{x}} \cdot\left(\rho_{\mathrm{f}} \phi \underline{v}_{\mathrm{r}}^{*}\right)+\phi \underline{\underline{\sigma}}_{\mathrm{vis}}: \underline{\underline{\varepsilon}}\left(\underline{v}_{\mathrm{s}}^{*}\right)\right) \mathrm{d} \Omega \\
-\int_{\Omega^{t}} \rho_{\mathrm{f}} \phi \underline{f} \cdot \underline{v}_{\mathrm{s}}^{*} \mathrm{~d} \Omega-\int_{\Gamma_{N}^{t}} \phi \underline{t}^{\prime} \cdot \underline{v}_{\mathrm{s}}^{*} d S-\int_{\Gamma_{N \text { nof }}^{t}} \phi\left(\pi_{\tau} \underline{\underline{t}}\right) \cdot \underline{v}_{\mathrm{s}}^{*} d S .
\end{array}
$$

In the above System $(5)$, the test functions $\left(\underline{v}_{\mathrm{s}}^{*}, \underline{v}_{\mathrm{r}}^{*}\right)$ are assumed to satisfy homogeneous Dirichlet boundary conditions on $\Gamma_{D}^{0}\left(\right.$ or $\left.\Gamma_{D}^{t}\right)$. Moreover, $\underline{v}_{\mathrm{r}}^{*}$ must satisfy $\left.\underline{v}_{\mathrm{r}}^{*}\right|_{\Gamma_{N \text { nos }}^{t}}=0$ and $\left.\underline{v}_{\mathrm{r}}^{*} \cdot \underline{n}\right|_{\Gamma_{N \mathrm{nof}}^{t}}=0$, in adequation with the fluid-solid kinematical coupling conditions. Note that we remain formal at this stage, deferring the specific definitions of the mathematical spaces to the mathematical analysis of the linearized equations in Section 2.3.

\subsection{Linearized formulation}

We consider a linearization of (5) around the solution at rest, namely $\left(\underline{u}_{\mathrm{s}}, \underline{\bar{v}}_{\mathrm{s}}, \overline{\mathrm{v}}_{\mathrm{f}}, \bar{m}\right) \equiv 0$ (then $\bar{p} \equiv 0)$. Note that $\phi$ will vary around $\phi_{0}=\bar{\phi} \neq 0$. In the linearization process, $\Omega^{t}$ reduces to $\Omega^{0}$, hence we drop the 0 superscript in all the geometric quantities to simplify the notation. Moreover, we do not need to distinguish between $\underline{\xi}$ and $\underline{x}$ for spatial derivatives, nor between Cauchy and second Piola-Kirchhoff stresses, and the Green-Lagrange strain tensor $\underline{\underline{e}}$ reduces to 
the linearized elasticity tensor $\underline{\underline{\varepsilon}}$. Retaining only the first-order terms, System (5) then directly reduces to

$$
\begin{aligned}
& \iint_{\Omega} \rho_{\mathrm{s}}(1-\bar{\phi}) \partial_{t} \underline{v}_{\mathrm{s}} \cdot \underline{v}_{\mathrm{s}}^{*} \mathrm{~d} \Omega+\int_{\Omega} \underline{\sigma}_{\mathrm{s}}: \underline{\underline{\varepsilon}}\left(\underline{v}_{\mathrm{s}}^{*}\right) \mathrm{d} \Omega-\int_{\Omega}\left(\underline{v}_{\mathrm{f}}-\underline{v}_{\mathrm{s}}\right) \cdot \bar{\phi}^{2} \underline{k}_{\mathrm{f}}^{-1} \cdot \underline{v}_{\mathrm{s}}^{*} \mathrm{~d} \Omega \\
& +\int_{\Omega} p \underline{\nabla} \bar{\phi} \cdot \underline{v}_{\mathrm{s}}^{*} \mathrm{~d} \Omega=\int_{\Omega} \rho_{s}(1-\bar{\phi}) \underline{f} \cdot \underline{v}_{\mathrm{s}}^{*} \mathrm{~d} \Omega+\int_{\Gamma_{N}}(1-\bar{\phi}) \underline{t} \cdot \underline{v}_{\mathrm{s}}^{*} d S \\
& +\int_{\Gamma_{N \mathrm{nof}} \cup \Gamma_{N \mathrm{nos}}} \underline{t} \cdot \underline{v}_{\mathrm{s}}^{*} d S-\int_{\Gamma_{N \mathrm{nof}}} \bar{\phi}\left(\pi_{\tau} \underline{t}\right) \cdot \underline{v}_{\mathrm{s}}^{*} d S-\mathcal{R}_{\mathrm{f}}^{\mathrm{c}, \operatorname{lin}}\left(\underline{v}_{\mathrm{s}}^{*}\right) \\
& \int_{\Omega} \rho_{\mathrm{f}} \bar{\phi} \partial_{t} \underline{v}_{\mathrm{f}} \cdot \underline{v}_{\mathrm{r}}^{*} \mathrm{~d} \Omega+\int_{\Omega}\left(-p \underline{\nabla} \cdot\left(\bar{\phi} \underline{v}_{\mathrm{r}}^{*}\right)+\bar{\phi} \underline{\underline{\sigma}}_{\mathrm{vis}}\left(\underline{v}_{\mathrm{f}}\right): \underline{\underline{\varepsilon}}\left(\underline{v}_{\mathrm{r}}^{*}\right)\right) \mathrm{d} \Omega \\
& +\int_{\Omega}\left(\underline{v}_{\mathrm{f}}-\underline{v}_{\mathrm{s}}\right) \cdot \bar{\phi}^{2} \underline{\underline{k}}_{\mathrm{f}}^{-1} \cdot \underline{v}_{\mathrm{r}}^{*} \mathrm{~d} \Omega=\int_{\Omega} \rho_{\mathrm{f}} \bar{\phi} \underline{f} \cdot \underline{v}_{\mathrm{r}}^{*} \mathrm{~d} \Omega \\
& +\int_{\Gamma_{N}} \bar{\phi} \underline{t} \cdot \underline{v}_{\mathrm{r}}^{*} d S+\int_{\Gamma_{N \mathrm{nof}}} \bar{\phi}\left(\pi_{\tau} \underline{t}\right) \cdot \underline{v}_{\mathrm{r}}^{*} d S \\
& \left(\int_{\Omega} \partial_{t} m p^{*} \mathrm{~d} \Omega+\int_{\Omega} \rho_{\mathrm{f}} \underline{\nabla} \cdot\left(\bar{\phi}\left(\underline{v}_{\mathrm{f}}-\underline{v}_{\mathrm{S}}\right)\right) p^{*} \mathrm{~d} \Omega=\int_{\Omega} \theta p^{*} \mathrm{~d} \Omega,\right.
\end{aligned}
$$

with

$$
\begin{aligned}
\mathcal{R}_{\mathrm{f}}^{\mathrm{c}, \operatorname{lin}}\left(\underline{v}_{\mathrm{s}}^{*}\right) & =\int_{\Omega} \rho_{\mathrm{f}} \bar{\phi} \partial_{t} \underline{v}_{\mathrm{f}} \cdot \underline{v}_{\mathrm{s}}^{*} \mathrm{~d} \Omega \\
+ & \left.\int_{\Omega}\left(-p \underline{\nabla} \cdot\left(\bar{\phi} \underline{v}_{\mathrm{r}}^{*}\right)+\bar{\phi} \underline{\underline{\sigma}}_{\mathrm{vis}}\left(\underline{v}_{\mathrm{f}}\right): \underline{\varepsilon}_{\underline{v}} \underline{v}_{\mathrm{s}}^{*}\right)\right) \mathrm{d} \Omega+\int_{\Omega}\left(\underline{v}_{\mathrm{f}}-\underline{v}_{\mathrm{s}}\right) \cdot \bar{\phi}^{2} \underline{\underline{k}}_{\mathrm{f}}^{-1} \cdot \underline{v}_{\mathrm{s}}^{*} \mathrm{~d} \Omega \\
& \quad-\int_{\Omega} \rho_{\mathrm{f}} \bar{\phi} \underline{f} \cdot \underline{v}_{\mathrm{s}}^{*} \mathrm{~d} \Omega-\int_{\Gamma_{N}} \bar{\phi} \underline{t} \cdot \underline{v}_{\mathrm{s}}^{*} d S-\int_{\Gamma_{N \text { nof }}} \bar{\phi}\left(\pi_{\tau}\right) \cdot \underline{v}_{\mathrm{s}}^{*} d S .
\end{aligned}
$$

In order to complete the derivation of the linearized formulation, we need to specify and also linearize the constitutive equations. As proposed in [6], we consider the solid free energy in the additive form

$$
\Psi_{\mathrm{s}}\left(\underline{\underline{e}}, J_{\mathrm{s}}\right)=W_{\text {skel }}(\underline{\underline{e}})+W_{\text {bulk }}\left(J_{\mathrm{s}}\right),
$$

with the simplest possible expression for each part, namely

$$
\left\{\begin{array}{c}
W_{\text {skel }}(\underline{\underline{e}})=\frac{\lambda}{2}(\operatorname{tr} \underline{\underline{e}})^{2}+\mu \underline{\underline{e}}: \underline{\underline{e}}, \\
W_{\text {bulk }}\left(J_{\mathrm{s}}\right)=\frac{\kappa_{\mathrm{s}}}{2}\left(\frac{J_{\mathrm{s}}}{1-\phi_{0}}-1\right)^{2} .
\end{array}\right.
$$

Note that $W_{\text {skel }}(\underline{\underline{e}})$ is the Saint-Venant-Kirchhoff hyperelastic potential, namely, the direct extension of linear isotropic elasticity to large displacements mechanics. Finally, we choose $\Psi_{v}(\underline{\underline{\dot{e}}})=\mu_{\mathrm{s}} \underline{\underline{\dot{e}}}: \underline{\underline{\dot{e}}}$, and $(2)$ gives

$$
\underline{\underline{\Sigma}}_{\mathrm{s}}=\lambda(\operatorname{tr} \underline{\underline{e}}) \underline{\underline{\mathbb{1}}}+2 \mu \underline{\underline{e}}+2 \mu_{\mathrm{s}} \underline{\underline{\underline{e}}}-(1-\phi) p J \underline{\underline{C}}^{-1},
$$

for which the linearized counterpart reads

$$
\underline{\underline{\sigma}}_{\mathrm{S}}=\underline{\underline{\sigma}}_{\mathrm{skel}}\left(\underline{u}_{\mathrm{S}}\right)+2 \mu_{\mathrm{S}} \underline{\underline{\varepsilon}}\left(\underline{v}_{\mathrm{S}}\right)-(1-\bar{\phi}) p \underline{\underline{\underline{\mathbb{1}}}},
$$


with

$$
\underline{\sigma}_{\text {skel }}\left(\underline{u}_{\mathrm{s}}\right)=\lambda \operatorname{tr} \underline{\underline{\varepsilon}}\left(\underline{u}_{\mathrm{s}}\right) \underline{\underline{\mathbb{1}}}+2 \mu \underline{\underline{\varepsilon}}\left(\underline{u}_{\mathrm{s}}\right) .
$$

Likewise, the pressure law is obtained by applying (3), viz.

$$
p=\frac{\kappa_{\mathrm{s}}}{1-\phi_{0}}\left(1-\frac{J_{\mathrm{s}}}{1-\phi_{0}}\right)=\frac{\kappa_{\mathrm{s}}}{\left(1-\phi_{0}\right)^{2}}\left(1-J+\frac{m}{\rho_{\mathrm{f}}}\right),
$$

and the linearized form is obtained by directly transforming $J-1$ into $\underline{\nabla} \cdot \underline{u}_{\mathrm{s}}$, i.e.

$$
p=\frac{\kappa_{\mathrm{S}}}{(1-\bar{\phi})^{2}}\left(\frac{m}{\rho_{\mathrm{f}}}-\underline{\nabla} \cdot \underline{u}_{\mathrm{s}}\right) .
$$

Using this equation to eliminate $m$ in (8c), we finally obtain the alternative form

$$
\int_{\Omega}\left(\frac{(1-\bar{\phi})^{2}}{\kappa_{\mathrm{s}}} \partial_{t} p+\underline{\nabla} \cdot \underline{v}_{\mathrm{s}}\right) p^{*} \mathrm{~d} \Omega+\int_{\Omega} \underline{\nabla} \cdot\left(\bar{\phi}\left(\underline{v}_{\mathrm{f}}-\underline{v}_{\mathrm{s}}\right)\right) p^{*} \mathrm{~d} \Omega=\int_{\Omega} \frac{\theta}{\rho_{\mathrm{f}}} p^{*} \mathrm{~d} \Omega .
$$

Note that the boundary conditions are directly inferred from those defined in the nonlinear setting, so we do not rephrase them for the sake of brevity.

The corresponding strong formulation reads

$$
\left\{\begin{array}{l}
\rho_{\mathrm{s}}(1-\bar{\phi}) \partial_{t} \underline{v}_{\mathrm{s}}-\underline{\operatorname{div}} \underline{\underline{\sigma}}-\bar{\phi}_{\mathrm{s}}^{2} \underline{\underline{k}}_{\mathrm{f}}^{-1} \cdot\left(\underline{v}_{\mathrm{f}}-\underline{v}_{\mathrm{s}}\right)+p \underline{\nabla} \bar{\phi}=\rho_{s}(1-\bar{\phi}) \underline{f} \\
\rho_{\mathrm{f}} \bar{\phi} \partial_{t} \underline{v}_{\mathrm{f}}+\bar{\phi} \underline{\nabla} p-\underline{\operatorname{div}}\left(\bar{\phi} \underline{\sigma}_{\mathrm{vis}}\right)+\bar{\phi}^{2} \underline{\underline{k}}_{\mathrm{f}}^{-1} \cdot\left(\underline{v}_{\mathrm{f}}-\underline{v}_{\mathrm{s}}\right)=\rho_{\mathrm{f}} \bar{\phi} \underline{f} \\
\frac{(1-\bar{\phi})^{2}}{\kappa_{\mathrm{s}}} \partial_{t} p+\underline{\nabla} \cdot\left((1-\bar{\phi}) \underline{v}_{\mathrm{s}}+\bar{\phi} \underline{v}_{\mathrm{f}}\right)=\frac{\theta}{\rho_{\mathrm{f}}}
\end{array}\right.
$$

inside the domain, with the same boundary conditions. We point out that the constraint that prevails in the case of solid incompressibility $\left(\kappa_{\mathrm{S}} \rightarrow+\infty\right)$ - i.e. complete incompressibility, since we already assumed the fluid to be incompressible - is

$$
\underline{\nabla} \cdot\left((1-\bar{\phi}) \underline{v}_{\mathrm{s}}+\bar{\phi} \underline{v}_{\mathrm{f}}\right)=\frac{\theta}{\rho_{\mathrm{f}}} .
$$

\subsection{Solution existence}

We now need to specify the spaces of existence of the solution of the above linearized equations in adequate functional spaces. First, let us introduce $\mathcal{Q}^{d}=L^{2}(\Omega)^{d}$ and $\mathcal{U}=H^{1}(\Omega)^{3}$. Then, for any $\underline{u}^{\mathrm{pr}} \in H^{\frac{1}{2}}\left(\Gamma_{D}\right)$, we define

$$
\mathcal{U}_{\Gamma_{D}}\left(\underline{u}^{\mathrm{pr}}\right)=\left\{\underline{u} \in \mathcal{U}|\underline{u}|_{\Gamma_{D}}=\underline{u}^{\mathrm{pr}}\right\} .
$$

We also assume that $\bar{\phi} \in C^{1}(\bar{\Omega})$, and that there exist two constants $\phi_{\min }$ and $\phi_{\max }$ such that

$$
0<\phi_{\min } \leq \bar{\phi} \leq \phi_{\max }<1,
$$

which is physically justified, indeed. Therefore, for any function $\rho_{\mathrm{f}}$ and $\rho_{\mathrm{s}}$ bounded from below - strictly positively - and above, the quantities

$$
\forall \underline{v} \in \mathcal{Q}^{3}, \quad\|\underline{v}\|_{\Omega, \rho_{\mathrm{f}} \bar{\phi}}^{2}=\int_{\Omega} \rho_{\mathrm{f}} \bar{\phi}\|\underline{v}\|^{2} \mathrm{~d} x, \quad\|\underline{v}\|_{\Omega, \rho_{\mathrm{s}}(1-\bar{\phi})}^{2}=\int_{\Omega} \rho_{\mathrm{S}}(1-\bar{\phi})\|\underline{v}\|^{2} \mathrm{~d} \Omega
$$

define two equivalent norms on $\mathcal{Q}^{3}$. Similarly, we introduce the norm on $L^{2}(\Omega)$

$$
\forall p \in \mathcal{Q}, \quad\|p\|_{\Omega,(1-\bar{\phi})^{2}}^{2}=\int_{\Omega}(1-\bar{\phi})^{2} p^{2} \mathrm{~d} \Omega .
$$


Moreover, assuming that the Lamé coefficients $(\lambda, \mu)$ are bounded from below - positively for $\lambda$ and strictly positively for $\mu$ - we can define in $\mathcal{U}_{\Gamma_{D}}\left(\underline{u}^{\mathrm{pr}}\right)$ the scalar product

$$
\left(\underline{u}_{\mathrm{s}}, \underline{u}^{*}\right)_{\text {skel }}=\int_{\Omega} \underline{\sigma}_{\text {skel }}\left(\underline{u}_{\mathrm{s}}\right): \underline{\underline{\varepsilon}}\left(\underline{u}_{\mathrm{s}}^{*}\right) d \Omega,
$$

for which the associated norm is equivalent to the usual $H^{1}$-norm due to Korn inequality [7]. We then define the affine state space

$$
\mathcal{X}=\left\{X=\left(\underline{u}_{\mathrm{s}}, \underline{v}_{\mathrm{s}}, \underline{v}_{\mathrm{f}}, p\right) \in \mathcal{U}_{\Gamma_{D}}\left(\underline{u}_{\mathrm{s}}^{\mathrm{pr}}\right) \times \mathcal{Q}^{3} \times \mathcal{Q}^{3} \times \mathcal{Q}\right\},
$$

and, typically for regular enough data, we seek a solution of $(8 \mathrm{a})-(8 \mathrm{~b})-(15)$ in $L^{2}((0, T), \mathcal{X})$ or $C((0, T) ; \mathcal{X})$.

In order to give an example of such an existence result consistent with our further numerical analysis, we restrict ourself to a simpler case where we have homogeneous Dirichlet boundary conditions. We then introduce $\mathcal{X}^{0}$ the Hilbert space associated with homogeneous Dirichlet boundary conditions that we equip with the energy norm

$$
\|X\|_{\mathcal{E}}^{2}=\frac{1}{2}\left\|\underline{u}_{\mathrm{s}}\right\|_{\text {skel }}^{2}+\frac{1}{2}\left\|\underline{v}_{\mathrm{s}}\right\|_{\Omega, \rho_{\mathrm{s}}(1-\bar{\phi})}^{2}+\frac{1}{2}\left\|\underline{v}_{\mathrm{f}}\right\|_{\Omega, \rho_{\mathrm{f}} \bar{\phi}}^{2}+\frac{1}{2 \kappa_{\mathrm{s}}}\|p\|_{\Omega,(1-\bar{\phi})^{2}}^{2},
$$

with $(\cdot, \cdot)_{\mathcal{E}}$ the associated scalar product. Moreover, we introduce the space $\mathcal{V}^{0} \subset \mathcal{X}^{0}$ defined as

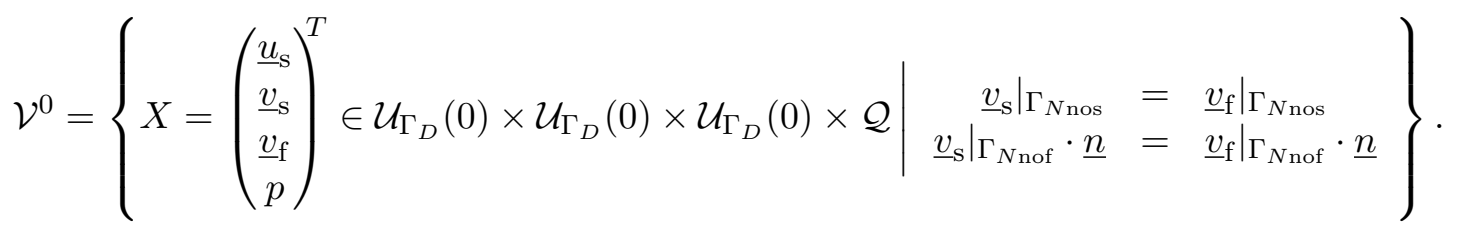

Note that we have $\mathcal{V}^{0} \subset \mathcal{X}^{0} \equiv \mathcal{X}^{0^{\prime}} \subset \mathcal{V}^{0^{\prime}}$ with continuous injection.

We now want to rewrite our problem in a single variational equation. To that purpose, we first write the relationship between displacement and velocity in a weak form, namely

$$
\forall \underline{u}_{\mathrm{s}}^{*} \in \mathcal{U}(0), \quad \int_{\Omega} \underline{\sigma}_{\text {skel }}\left(\partial_{t} \underline{u}_{\mathrm{s}}\right): \underline{\underline{\varepsilon}}\left(\underline{u}_{\mathrm{s}}^{*}\right) \mathrm{d} \Omega=\int_{\Omega} \underline{\sigma}_{\text {skel }}\left(\underline{v}_{\mathrm{s}}\right): \underline{\underline{\varepsilon}}\left(\underline{u}_{\mathrm{s}}^{*}\right) \mathrm{d} \Omega
$$

and we then add this equation together with (8a), (8b) and (15), with the substitution $\underline{v}_{\mathrm{r}}^{*}=$ $\underline{v}_{\mathrm{f}}^{*}-\underline{v}_{\mathrm{s}}^{*}$. After some simplifications, we obtain the formal variational equation

$$
\forall X^{*} \in \mathcal{V}^{0}, \quad\left(\frac{\mathrm{d} X}{\mathrm{dt}}(t), X^{*}\right)_{\mathcal{E}}+M\left(X(t), X^{*}\right)=L\left(X^{*}\right),
$$

where the bilinear form $M$ is defined in $\mathcal{V}^{0}$ by

$$
\begin{aligned}
& M\left(X, X^{*}\right)=- \int_{\Omega} \underline{\sigma}_{\mathrm{skel}}\left(\underline{v}_{\mathrm{s}}\right): \underline{\underline{\varepsilon}}\left(\underline{u}_{\mathrm{s}}^{*}\right) \mathrm{d} \Omega+\int_{\Omega} \underline{\underline{\sigma}}_{\mathrm{skel}}\left(\underline{u}_{\mathrm{s}}\right): \underline{\underline{\varepsilon}}\left(\underline{v}_{\mathrm{s}}^{*}\right) \mathrm{d} \Omega+2 \int_{\Omega} \mu_{\mathrm{s}} \underline{\underline{\varepsilon}}\left(\underline{v}_{\mathrm{s}}\right): \underline{\underline{\varepsilon}}\left(\underline{v}_{\mathrm{s}}^{*}\right) \mathrm{d} \Omega \\
&-\int_{\Omega}(1-\bar{\phi}) p \underline{\nabla} \cdot \underline{v}_{\mathrm{s}}^{*} \mathrm{~d} \Omega \\
&+\int_{\Omega}\left(\underline{v}_{\mathrm{f}}-\underline{v}_{\mathrm{s}}\right) \cdot \bar{\phi}^{2} \underline{\underline{k}}_{\mathrm{f}}^{-1} \cdot\left(\underline{v}_{\mathrm{f}}^{*}-\underline{v}_{\mathrm{s}}^{*}\right) \mathrm{d} \Omega \\
&+\int_{\Omega} p \underline{\nabla} \bar{\phi} \cdot \underline{v}_{\mathrm{s}}^{*} \mathrm{~d} \Omega+\int_{\Omega}\left(-p \underline{\nabla} \cdot\left(\bar{\phi} \underline{v}_{\mathrm{f}}^{*}\right)+\bar{\phi} \underline{\underline{\sigma}}_{\mathrm{vis}}\left(\underline{v}_{\mathrm{f}}\right): \underline{\underline{\varepsilon}}\left(\underline{v}_{\mathrm{f}}^{*}\right)\right) \mathrm{d} \Omega \\
&+\int_{\Omega}\left(\underline{\nabla} \cdot \underline{v}_{\mathrm{s}}\right) p^{*} \mathrm{~d} \Omega+\int_{\Omega} \underline{\nabla} \cdot\left(\bar{\phi}\left(\underline{v}_{\mathrm{f}}-\underline{v}_{\mathrm{s}}\right)\right) p^{*} \mathrm{~d} \Omega,
\end{aligned}
$$


and the linear form $L$ is given by

$$
\begin{array}{r}
L\left(X^{*}\right)=\int_{\Omega} \rho_{s}(1-\bar{\phi}) \underline{f} \cdot \underline{v}_{\mathrm{s}}^{*} d \Omega+\int_{\Gamma_{N}}(1-\bar{\phi}) \underline{t} \cdot \underline{v}_{\mathrm{s}}^{*} d S+\int_{\Gamma_{N \mathrm{nof}} \cup \Gamma_{N \mathrm{nos}}} \underline{t} \cdot \underline{v}_{\mathrm{s}}^{*} d S-\int_{\Gamma_{N \text { nof }}} \bar{\phi}\left(\pi_{\tau} \underline{t}\right) \cdot \underline{v}_{\mathrm{s}}^{*} d S \\
+\int_{\Omega} \rho_{\mathrm{f}} \bar{\phi} \underline{f} \cdot \underline{v}_{\mathrm{f}}^{*} d \Omega+\int_{\Gamma_{N} \cup \Gamma_{N \mathrm{nof}}} \bar{\phi} \cdot \underline{v}_{\mathrm{f}}^{*} d S+\int_{\Omega} \theta p^{*} d \Omega,
\end{array}
$$

Note that, with a more condensed notation, (20) also reads

$$
\begin{aligned}
M\left(X, X^{*}\right)= & -\left(\underline{v}_{\mathrm{s}}, \underline{u}^{*}\right)_{\text {skel }}+\left(\underline{u}_{\mathrm{s}}, \underline{v}_{\mathrm{s}}^{*}\right)_{\mathrm{skel}}+2\left(\underline{\underline{\varepsilon}}\left(\underline{v}_{\mathrm{s}}\right), \underline{\underline{\varepsilon}}\left(\underline{v}_{\mathrm{s}}^{*}\right)\right)_{\mu_{\mathrm{s}}, \Omega} \\
& -\left(p, \operatorname{div}\left(\bar{\phi} \underline{v}_{\mathrm{f}}^{*}+(1-\bar{\phi}) \underline{v}_{\mathrm{s}}^{*}\right)\right)_{L^{2}(\Omega)}+\left(p^{*}, \operatorname{div}\left(\bar{\phi} \underline{v}_{\mathrm{f}}+(1-\bar{\phi}) \underline{v}_{\mathrm{s}}\right)\right)_{L^{2}(\Omega)} \\
& +2\left(\underline{\underline{\varepsilon}}\left(\underline{v}_{\mathrm{f}}\right), \underline{\underline{\varepsilon}}\left(\underline{v}_{\mathrm{f}}^{*}\right)\right)_{\bar{\phi} \mu_{\mathrm{f}}, \Omega}+\left(\underline{v}_{\mathrm{f}}-\underline{v}_{\mathrm{s}}, \underline{v}_{\mathrm{f}}^{*}-\underline{v}_{\mathrm{s}}^{*}\right)_{\bar{\phi}^{2} \underline{k}_{\mathrm{f}}^{-1}, \Omega} .
\end{aligned}
$$

\section{Proposition 1}

The bilinear form $M$ is $\mathcal{V}^{0}-\mathcal{X}^{0}$ coercive, namely, there exist $\alpha>0$ and $\lambda \in \mathbb{R}$ such that

$$
\forall X \in \mathcal{V}^{0}, \quad M(X, X)+\lambda\|X\|_{\mathcal{E}}^{2} \geq \alpha\|X\|_{\mathcal{V}^{0}}^{2} .
$$

Proof. We have

$$
M(X, X)=2\left(\underline{\underline{\varepsilon}}\left(\underline{v}_{\mathrm{s}}\right), \underline{\underline{\varepsilon}}\left(\underline{v}_{\mathrm{s}}\right)\right)_{\mu_{\mathrm{s}}, \Omega}+2\left(\underline{\underline{\varepsilon}}\left(\underline{v}_{\mathrm{f}}\right), \underline{\underline{\varepsilon}}\left(\underline{v}_{\mathrm{f}}\right)\right)_{\bar{\phi} \mu_{\mathrm{f}}, \Omega}+\left(\underline{v}_{\mathrm{f}}-\underline{v}_{\mathrm{s}}, \underline{v}_{\mathrm{f}}-\underline{v}_{\mathrm{s}}\right)_{\bar{\phi}^{2} \underline{\underline{k}}_{\mathrm{f}}^{-1}, \Omega} .
$$

Therefore, for all $\lambda>0$, the bilinear form $M_{\lambda}\left(X, X^{*}\right)=M\left(X, X^{*}\right)+\lambda\left(X, X^{*}\right)_{\mathcal{E}}$ is $\mathcal{V}^{0}$-coercive.

Considering now that the initial condition $X(0) \in \mathcal{X}$ and assuming that the data are regular enough so that $L \in L^{2}\left(0, T, \mathcal{V}^{0^{\prime}}\right)$, we have for all $T$ - see [15, Vol. 1, Chap. 3, §4, Theorem 4.1], and also [1, II-2, Theorem 1.1] - a unique solution in

$$
\mathcal{W}^{0}(0, T)=\left\{X \in L^{2}\left(0, T ; \mathcal{V}^{0}\right) ; \quad \frac{\mathrm{d} X}{\mathrm{dt}} \in L^{2}\left(0, T ; \mathcal{V}^{0^{\prime}}\right)\right\},
$$

of the dynamics

$$
\left\{\begin{array}{l}
\left\langle\frac{\mathrm{d} X}{\mathrm{dt}}(t), X^{*}\right\rangle_{\mathcal{V}^{0^{\prime}}, \mathcal{V}^{0}}+M\left(X(t), X^{*}\right)=L\left(X^{*}\right), \quad \text { in } L^{2}(0, T) \\
X(0)=X_{0},
\end{array}\right.
$$

where the first term should be understood as

$$
\left\langle\frac{\mathrm{d} X}{\mathrm{dt}}(t), X^{*}\right\rangle_{\mathcal{V}^{0^{\prime}, \mathcal{V}^{0}}}=\left(\frac{\mathrm{d} X}{\mathrm{dt}}(t), X^{*}\right)_{\mathcal{E}}
$$

if $\frac{\mathrm{d} X}{\mathrm{dt}} \in \mathcal{X}$ is regular enough, since we have chosen $\mathcal{X}$ as the pivot space.

Note finally that we have a continuous injection $\mathcal{W}^{0}(0, T) \hookrightarrow C\left([0, T] ; \mathcal{X}^{0}\right)[15$, Vol. 1 , Prop 2.1 and Thm 3.1], hence the solution can also be understood in the sense of the Duhamel formula

$$
\forall t \in[0, T], \quad X(t)=\Phi(t) X(0)+\int_{0}^{t} \Phi(t-s) r(s) \mathrm{d} s,
$$

where $\Phi$ is the evolution operator associated with our problem.

To conclude this section, let us mention that the case where we seek solutions with nonhomogeneous boundary conditions in $\mathcal{X}$ is more technical to write - albeit similar in essence, as usual - since we need to define adequate liftings of the boundary conditions. 


\subsection{Space-discretized formulation}

We will use a finite element strategy, and we accordingly consider a mesh subdividing the domain $\Omega$, parametrized by the quantity $h$ representing the maximum diameter of all the elements in the mesh. We assume this mesh to be strictly geometrically conforming - i.e. the external boundaries are exactly those of $\Omega$ - to keep away from the technicalities induced by geometric consistency errors in the analysis. We also assume that the solid displacement and the velocity are discretized in the same space $\mathcal{W}_{h}^{s}$, the fluid velocity is chosen in $\mathcal{W}_{h}^{f}$ and the pressure in $\mathcal{Q}_{h}$. For any $\underline{u}^{\mathrm{pr}} \in \mathcal{W}_{h}^{f}$, we define

$$
\mathcal{W}_{h}^{f}\left(\underline{u}^{\mathrm{pr}}\right)=\left\{\underline{u} \in \mathcal{W}_{h}^{f}|\underline{u}|_{\Gamma_{D}}=\left.\underline{u}^{\mathrm{pr}}\right|_{\Gamma_{D}}\right\},
$$

with a similar notation for $\mathcal{W}_{h}^{s}$. Then the finite element spaces associated with $\mathcal{V}$ and $\mathcal{V}^{0}$ are

$$
\begin{aligned}
& \mathcal{V}_{h}=\mathcal{W}_{h}^{s}\left(\underline{u}_{\mathrm{s}, h}^{\mathrm{pr}}\right) \times \mathcal{W}_{h}^{s}\left(\partial_{t} \underline{u}_{\mathrm{s}, h}^{\mathrm{pr}}\right) \times \mathcal{W}_{h}^{f}\left(\underline{v}_{\mathrm{f}, h}^{\mathrm{pr}}\right) \times \mathcal{Q}_{h}, \\
& \mathcal{V}_{h}^{0}=\mathcal{W}_{h}^{s}(0) \times \mathcal{W}_{h}^{s}(0) \times \mathcal{W}_{h}^{f}(0) \times \mathcal{Q}_{h},
\end{aligned}
$$

for $\left(\underline{u}_{\mathrm{s}, h}^{\mathrm{pr}}, \underline{v}_{\mathrm{f}, h}^{\mathrm{pr}}\right)$ in $\mathcal{W}_{h}^{s} \times \mathcal{W}_{h}^{f}$.

As in the splitting time-scheme presented in a non-linear context in [5], fluid and solid constituents are implicitly and weakly coupled on $\Gamma_{N \text { nos }}$ and $\Gamma_{N \text { nof }}$ with Robin coupling conditions derived from Nitsche's interface method $[3,4,16]$. Unlike in $(8 \mathrm{~b})$, this condition is weakly imposed in the fluid resolution, so no Dirichlet boundary condition is enforced on the fluid test function $\underline{v}_{\mathrm{f}, h}^{*}$ on $\Gamma_{N \text { nos }}$ and $\Gamma_{N \text { nof }}$. Therefore, the integration by part that leads to $(8 \mathrm{~b})$ yields the new terms $\left(\underline{\underline{\sigma}}_{\mathrm{f}}\left(\underline{v}_{\mathrm{f}, h}, p_{h}\right) \cdot \underline{n}, \underline{v}_{\mathrm{f}, h}^{*}\right)_{\bar{\phi}, \Gamma_{N \mathrm{nos}}}+\left(\left(\underline{\underline{\sigma}}_{\mathrm{f}}\left(\underline{v}_{\mathrm{f}, h}, p_{h}\right) \cdot \underline{n}\right) \cdot \underline{n}, \underline{v}_{\mathrm{f}, h}^{*} \cdot \underline{n}\right)_{\bar{\phi}, \Gamma_{N \text { nof }}}$ in the fluid equation right-hand side (RHS), with $\underline{\underline{\sigma}}_{\mathrm{f}}\left(\underline{v}_{\mathrm{f}, h}, p_{h}\right)=\underline{\underline{\sigma}}_{\mathrm{vis}}\left(\underline{v}_{\mathrm{f}, h}\right)-p_{h} \underline{\underline{\mathbb{1}}}$. Furthermore, we use the identity $\mathcal{R}_{\mathrm{f}}^{\mathrm{c}, \mathrm{lin}}\left(\underline{v}_{\mathrm{s}, h}^{*}\right)=\left(\underline{\underline{\sigma}}_{\mathrm{f}}\left(\underline{v}_{\mathrm{s}, h}, p_{h}\right) \cdot \underline{n}, \underline{v}_{\mathrm{s}, h}^{*}\right)_{\bar{\phi}, \Gamma_{N \text { nos }}}+\left(\left(\underline{\underline{\sigma}}_{\mathrm{f}}\left(\underline{v}_{\mathrm{f}, h}, p_{h}\right) \cdot \underline{n}\right) \cdot \underline{n}, \underline{v}_{\mathrm{s}, h}^{*} \cdot \underline{n}\right)_{\bar{\phi}, \Gamma_{N \text { nof }}}$.

We thus derive the following weak form at the space-discretized level, namely, for any $X_{h}^{*}=$ $\left(\underline{v}_{\mathrm{f}, h}^{*}, \underline{v}_{\mathrm{s}, h}^{*}, \underline{u}_{\mathrm{s}, h}^{*}, p_{h}^{*}\right)$ in $\mathcal{V}_{h}^{0}$

$$
\begin{aligned}
&\left(\frac{\mathrm{d}}{\mathrm{dt}} X_{h}, X_{h}^{*}\right)_{\mathcal{E}}+M\left(X_{h}, X_{h}^{*}\right)+\frac{\gamma}{h}\left(\underline{v}_{\mathrm{f}, h}-\underline{v}_{\mathrm{s}, h}, \underline{v}_{\mathrm{f}, h}^{*}-\underline{v}_{\mathrm{s}, h}^{*}\right)_{\mu_{\mathrm{f}} \bar{\phi}, \Gamma_{N \mathrm{nos}}} \\
&+\frac{\gamma}{h}\left(\left(\underline{v}_{\mathrm{f}, h}-\underline{v}_{\mathrm{s}, h}\right) \cdot \underline{n},\left(\underline{v}_{\mathrm{f}, h}^{*}-\underline{v}_{\mathrm{s}, h}^{*}\right) \cdot \underline{n}\right)_{\mu_{\mathrm{f}} \bar{\phi}, \Gamma_{N \mathrm{nof}}}-\left(\underline{\underline{\sigma}}_{\mathrm{f}}\left(\underline{v}_{\mathrm{f}, h}, p_{h}\right) \cdot \underline{n}, \underline{v}_{\mathrm{f}, h}^{*}-\underline{v}_{\mathrm{s}, h}^{*}\right)_{\bar{\phi}, \Gamma_{N \mathrm{nos}}} \\
&-\left(\left(\underline{\underline{\sigma}}_{\mathrm{f}}\left(\underline{v}_{\mathrm{f}, h}, p_{h}\right) \cdot \underline{n}\right) \cdot \underline{n},\left(\underline{v}_{\mathrm{f}, h}^{*}-\underline{v}_{\mathrm{s}, h}^{*}\right) \cdot \underline{n}\right)_{\bar{\phi}, \Gamma_{N \mathrm{nof}}}-\left(\underline{v}_{\mathrm{f}, h}-\underline{v}_{\mathrm{s}, h}, \underline{\sigma}_{\mathrm{f}}\left(\underline{v}_{\mathrm{f}, h}^{*},-p_{h}^{*}\right) \cdot \underline{n}\right)_{\bar{\phi}, \Gamma_{N \mathrm{nos}}} \\
&-\left(\left(\underline{v}_{\mathrm{f}, h}-\underline{v}_{\mathrm{s}, h}\right) \cdot \underline{n},\left(\underline{\underline{\sigma}}_{\mathrm{f}}\left(\underline{v}_{\mathrm{f}, h}^{*},-p_{h}^{*}\right) \cdot \underline{n}\right) \cdot \underline{n}\right)_{\bar{\phi}, \Gamma_{N \mathrm{nof}}}=L\left(X_{h}^{*}\right) .
\end{aligned}
$$

Note that we have symmetrized - respectively skew-symmetrized - the formulation with respect to $\underline{v}_{\mathrm{f}, h}$ - respectively $p_{h}$ - by incorporating consistent terms (i.e. that vanish with the continuous solution) on the boundaries $\Gamma_{N \text { nos }}$ and $\Gamma_{N \text { nof. }}$. This variational equation can be rephrased as

$$
\left(\frac{\mathrm{d}}{\mathrm{dt}} X_{h}, X_{h}^{*}\right)_{\mathcal{E}}+M_{h}\left(X_{h}, X_{h}^{*}\right)=L\left(X_{h}^{*}\right)
$$


where we introduced

$$
\begin{aligned}
& M_{h}(\left.X_{h}, X_{h}^{*}\right)=M\left(X_{h}, X_{h}^{*}\right)+\frac{\gamma}{h}\left(\underline{v}_{\mathrm{f}, h}-\underline{v}_{\mathrm{s}, h}, \underline{v}_{\mathrm{f}, h}^{*}-\underline{v}_{\mathrm{s}, h}^{*}\right)_{\mu \bar{\phi}, \Gamma_{N \mathrm{nos}}} \\
&+ \frac{\gamma}{h}\left(\left(\underline{v}_{\mathrm{f}, h}-\underline{v}_{\mathrm{s}, h}\right) \cdot \underline{n},\left(\underline{v}_{\mathrm{f}, h}^{*}-\underline{v}_{\mathrm{s}, h}^{*}\right) \cdot \underline{n}\right)_{\mu \bar{\phi}, \Gamma_{N \mathrm{nof}}}-\left(\underline{\underline{\sigma}}_{\mathrm{f}}\left(\underline{v}_{\mathrm{f}, h}, p_{h}\right) \cdot \underline{n}, \underline{v}_{\mathrm{f}, h}^{*}-\underline{v}_{\mathrm{s}, h}^{*}\right)_{\bar{\phi}, \Gamma_{N \mathrm{nos}}} \\
&-\left(\left(\underline{\underline{\sigma}}_{\mathrm{f}}\left(\underline{v}_{\mathrm{f}, h}, p_{h}\right) \cdot \underline{n}\right) \cdot \underline{n},\left(\underline{v}_{\mathrm{f}, h}^{*}-\underline{v}_{\mathrm{s}, h}^{*}\right) \cdot \underline{n}\right)_{\bar{\phi}, \Gamma_{N \mathrm{nof}}}-\left(\underline{v}_{\mathrm{f}, h}-\underline{v}_{\mathrm{s}, h}, \underline{\underline{f}}_{\mathrm{f}}\left(\underline{v}_{\mathrm{f}, h}^{*},-p_{h}^{*}\right) \cdot \underline{n}\right)_{\bar{\phi}, \Gamma_{N \mathrm{nos}}} \\
&-\left(\left(\underline{v}_{\mathrm{f}, h}-\underline{v}_{\mathrm{s}, h}\right) \cdot \underline{n},\left(\underline{\underline{\sigma}}_{\mathrm{f}}\left(\underline{v}_{\mathrm{f}, h}^{*},-p_{h}^{*}\right) \cdot \underline{n}\right) \cdot \underline{n}\right)_{\bar{\phi}, \Gamma_{N \mathrm{nof}}} .
\end{aligned}
$$

Note that (24) comes down to a Cauchy problem, so existence and uniqueness of the solution in the finite dimensional space $\mathcal{V}_{h}$ is guaranteed.

We start by establishing a discrete energy balance when the RHS $L$ vanishes, which is not exactly standard here due to the presence of the Nitsche terms. To that purpose we will use the following inverse inequality which can be proven by classical scaling arguments under standard assumptions on the mesh.

\section{Lemma 2 (Trace inverse inequality)}

There exists a constant $C_{i e}$ that depends on $\Omega, \Gamma_{N \text { nos }}$ and the type of shape functions, such that

$$
\|\underline{\underline{\varepsilon}}(\underline{w}) \cdot \underline{n}\|_{\bar{\phi}, \Gamma_{N \text { nos }} \cup \Gamma_{N \text { nof }}}^{2} \leq \frac{C_{i e}}{h}\|\underline{\underline{\varepsilon}}(\underline{w})\|_{\bar{\phi}, \Omega}^{2}, \quad \forall \underline{w} \in \mathcal{W}_{h}^{f} .
$$

Let $X_{h}$ in $\mathcal{V}_{h}$ be the solution of (24). Denoting by $\mathcal{E}_{h}=\left\|X_{h}\right\|_{\mathcal{X}}^{2}$ the energy of $X_{h}$, the evaluation of (24) with the test function $X_{h}^{*}=X_{h}$ gives

$$
\begin{array}{r}
\frac{\mathrm{d}}{\mathrm{dt}} \mathcal{E}_{h}+2\left\|\underline{\underline{\varepsilon}}\left(\underline{v}_{\mathrm{f}, h}\right)\right\|_{\mu_{\mathrm{f}} \bar{\phi}, \Omega}^{2}+2\left\|\underline{\underline{\varepsilon}}_{\underline{\mathrm{v}}, h} \underline{\mathrm{v}}_{\mu_{\mathrm{s}, \Omega}}^{2}+\right\| \underline{v}_{\mathrm{f}, h}-\underline{v}_{\mathrm{s}, h}\left\|_{\bar{\phi}^{2} \underline{k}_{\mathrm{f}}^{-1}, \Omega}^{2}+\frac{\gamma \mu_{\mathrm{f}}}{h}\right\| \underline{v}_{\mathrm{f}, h}-\underline{v}_{\mathrm{s}, h} \|_{\bar{\phi}, \Gamma_{N \mathrm{nos}}}^{2} \\
+\frac{\gamma \mu_{\mathrm{f}}}{h}\left\|\left(\underline{v}_{\mathrm{f}, h}-\underline{v}_{\mathrm{s}, h}\right) \cdot \underline{n}\right\|_{\bar{\phi}, \Gamma_{N \mathrm{nof}}}^{2}-4 \mu_{\mathrm{f}}\left(\underline{\underline{\varepsilon}}\left(\underline{v}_{\mathrm{f}, h}\right) \cdot \underline{n}, \underline{v}_{\mathrm{f}, h}-\underline{v}_{\mathrm{s}, h}\right)_{\bar{\phi}, \Gamma_{N \mathrm{nos}}} \\
-4 \mu_{\mathrm{f}}\left(\left(\underline{\underline{\varepsilon}}\left(\underline{v}_{\mathrm{f}, h}\right) \cdot \underline{n}\right) \cdot \underline{n},\left(\underline{v}_{\mathrm{f}, h}-\underline{v}_{\mathrm{s}, h}\right) \cdot \underline{n}\right)_{\bar{\phi}, \Gamma_{N \mathrm{nof}}}=0 .
\end{array}
$$

Then, we use Young's inequality $(a, b) \leq \frac{1}{2 L}\|a\|^{2}+\frac{L}{2}\|b\|^{2}$, with $L$ homogeneous to a length here, and the inverse inequality (26), to get

$$
\begin{aligned}
& 4 \mu_{\mathrm{f}}\left(\underline{\underline{\varepsilon}}\left(\underline{v}_{\mathrm{f}, h}\right) \cdot \underline{n}, \underline{v}_{\mathrm{f}, h}-\underline{v}_{\mathrm{s}, h}\right)_{\bar{\phi}, \Gamma_{N \mathrm{nos}}}+4 \mu_{\mathrm{f}}\left(\left(\underline{\underline{\varepsilon}}\left(\underline{v}_{\mathrm{f}, h}\right) \cdot \underline{n}\right) \cdot \underline{n},\left(\underline{v}_{\mathrm{f}, h}-\underline{v}_{\mathrm{s}, h}\right) \cdot \underline{n}\right)_{\bar{\phi}, \Gamma_{N \mathrm{nof}}} \\
& \leq 2 \mu_{\mathrm{f}}\left[L\left\|\underline{\underline{\varepsilon}}\left(\underline{v}_{\mathrm{f}, h}\right) \cdot \underline{n}\right\|_{\bar{\phi}, \Gamma_{N \mathrm{nos}} \cup \Gamma_{N \mathrm{nof}}}^{2}+\frac{1}{L}\left\|\underline{v}_{\mathrm{f}, h}-\underline{v}_{\mathrm{s}, h}\right\|_{\bar{\phi}, \Gamma_{N \mathrm{nos}}}^{2}+\frac{1}{L}\left\|\left(\underline{v}_{\mathrm{f}, h}-\underline{v}_{\mathrm{s}, h}\right) \cdot \underline{n}\right\|_{\bar{\phi}, \Gamma_{N \mathrm{nof}}}^{2}\right] \\
& \leq 2 \mu_{\mathrm{f}} \frac{C_{i e} L}{h}\left\|\underline{\varepsilon}_{\underline{\varepsilon}}\left(\underline{v}_{\mathrm{f}, h}\right)\right\|_{\bar{\phi}, \Omega}^{2}+2 \frac{\mu_{\mathrm{f}}}{L}\left\|\underline{v}_{\mathrm{f}, h}-\underline{v}_{\mathrm{s}, h}\right\|_{\bar{\phi}, \Gamma_{N \mathrm{nos}}}^{2}+2 \frac{\mu_{\mathrm{f}}}{L}\left\|\left(\underline{v}_{\mathrm{f}, h}-\underline{v}_{\mathrm{s}, h}\right) \cdot \underline{n}\right\|_{\bar{\phi}, \Gamma_{\text {Nnof }}}^{2} .
\end{aligned}
$$

We end up with the following energy balance

$$
\begin{aligned}
\frac{\mathrm{d}}{\mathrm{dt}} \mathcal{E}_{h}+2 \mu_{\mathrm{f}}\left(1-\frac{C_{i e} L}{h}\right) \| & \left\|\underline{\underline{\varepsilon}}\left(\underline{v}_{\mathrm{f}, h}\right)\right\|_{\bar{\phi}, \Omega}^{2}+2\left\|\underline{\underline{\varepsilon}}\left(\underline{v}_{\mathrm{s}, h}\right)\right\|_{\mu_{\mathrm{s}}, \Omega}^{2}+\mu_{\mathrm{f}}\left(\frac{\gamma}{h}-\frac{2}{L}\right)\left\|\underline{v}_{\mathrm{f}, h}-\underline{v}_{\mathrm{s}, h}\right\|_{\bar{\phi}, \Gamma_{N \mathrm{nos}}}^{2} \\
& +\mu_{\mathrm{f}}\left(\frac{\gamma}{h}-\frac{2}{L}\right)\left\|\left(\underline{v}_{\mathrm{f}, h}-\underline{v}_{\mathrm{s}, h}\right) \cdot \underline{n}\right\|_{\bar{\phi}, \Gamma_{N \mathrm{nof}}}^{2}+\left\|\underline{v}_{\mathrm{f}, h}-\underline{v}_{\mathrm{s}, h}\right\|_{\bar{\phi}^{2} \underline{\underline{k}}_{\mathrm{f}}^{-1}, \Omega}^{2} \leq 0,
\end{aligned}
$$

which ensures the stability of semi-discrete solution $X_{h}$ provided that $\gamma \geq 2 C_{i e}$. 


\subsection{Fully-discrete formulation}

We consider in (24) a first-order backward Euler method in the fluid, for both $\underline{v}_{\mathrm{f}}$ and $p$ - to be as close as possible to the splitting scheme proposed in a non-linear context in [5] - and a midpoint scheme for the solid. Using the notations $a^{n+\frac{1}{2}} \hat{=} \frac{a^{n+1}+a^{n}}{2}$ and $D_{\tau} a^{n+\frac{1}{2}} \hat{=} \frac{a^{n+1}-a^{n}}{\Delta t}$, we look for $\left(\underline{u}_{\mathrm{s}, h}^{n}, \underline{v}_{\mathrm{s}, h}^{n}, \underline{v}_{\mathrm{f}, h}^{n}, p_{h}^{n}\right)_{n \geq 0}$ in $\mathcal{V}_{h}^{\mathbb{N}}$ such that, for any $n$ in $\mathbb{N}$ and $\left(\underline{u}_{\mathrm{s}, h}^{*}, \underline{v}_{\mathrm{s}, h}^{*}, \underline{v}_{\mathrm{f}, h}^{*}, p_{h}^{*}\right)$ in $\mathcal{V}_{h}^{0}$,

$$
\begin{array}{r}
\rho_{\mathrm{f}}\left(D_{\tau} \underline{v}_{\mathrm{f}, h}^{n+\frac{1}{2}}, \underline{v}_{\mathrm{f}, h}^{*}\right)_{\bar{\phi}, \Omega}+\rho_{\mathrm{s}}\left(D_{\tau} \underline{v}_{\mathrm{s}, h}^{n+\frac{1}{2}}, \underline{v}_{\mathrm{s}, h}^{*}\right)_{1-\bar{\phi}, \Omega}+\frac{1}{\kappa_{\mathrm{s}}}\left(D_{\tau} p_{h}^{n+\frac{1}{2}}, p_{h}^{*}\right)_{(1-\bar{\phi})^{2}, \Omega} \\
+\left(D_{\tau} \underline{u}_{\mathrm{s}, h}^{n+\frac{1}{2}}, \underline{u}_{\mathrm{s}, h}^{*}\right)_{\mathrm{skel}}+M_{h}\left(\left(\underline{u}_{\mathrm{s}, h}^{n+\frac{1}{2}}, \underline{v}_{\mathrm{s}, h}^{n+\frac{1}{2}}, \underline{v}_{\mathrm{f}, h}^{n+1}, p_{h}^{n+1}\right),\left(\underline{u}_{\mathrm{s}, h}^{*}, \underline{v}_{\mathrm{s}, h}^{*}, \underline{v}_{\mathrm{f}, h}^{*}, p_{h}^{*}\right)\right) \\
=L^{n+\frac{1}{2}}\left(X_{h}^{*}\right),
\end{array}
$$

denoting

$$
\begin{aligned}
& L^{n+\frac{1}{2}}\left(X_{h}^{*}\right)=\int_{\Omega} \rho_{s}(1-\bar{\phi}) \underline{f}^{n+\frac{1}{2}} \cdot \underline{v}_{\mathrm{s}, h}^{*} d \Omega+\int_{\Gamma_{N}}(1-\bar{\phi}) \underline{t}^{n+\frac{1}{2}} \cdot \underline{v}_{\mathrm{s}, h}^{*} d S+\int_{\Gamma_{N \mathrm{nof}} \cup \Gamma_{N \mathrm{nos}}} \underline{t}^{n+\frac{1}{2}} \cdot \underline{v}_{\mathrm{s}, h}^{*} d S \\
& -\int_{\Gamma_{N \text { nof }}} \bar{\phi}\left(\pi_{\tau} \underline{t}^{n+\frac{1}{2}}\right) \cdot \underline{v}_{\mathrm{s}, h}^{*} d S+\int_{\Omega} \rho_{\mathrm{f}} \bar{\phi} \underline{f}^{n+\frac{1}{2}} \cdot \underline{v}_{\mathrm{f}, h}^{*} d \Omega+\int_{\Gamma_{N} \cup \Gamma_{N \text { nof }}} \bar{\phi} \underline{t}^{n+\frac{1}{2}} \cdot \underline{v}_{\mathrm{f}, h}^{*} d S+\int_{\Omega} \theta^{n+\frac{1}{2}} p_{h}^{*} d \Omega,
\end{aligned}
$$

and with adequate initial conditions.

\section{$3 \quad$ Numerical analysis}

In this section, we provide a detailed mathematical analysis of our proposed discretization procedure. Note that, as is quite usual in this context, we use the symbol $C$ to denote a generic positive constant that may take different values in successive occurrences. Likewise, $\alpha$ is used to denote a generic strictly positive constant. Throughout this section, we will assume $\underline{u}_{\mathrm{s}}^{\mathrm{pr}}=\underline{0}$ and $\underline{v}_{\mathrm{f}}^{\mathrm{pr}}=\underline{0}$ to avoid the cumbersome technicalities of non-homogeneous Dirichlet boundary conditions.

\subsection{Stability analysis in the energy norm}

In this specific section we will assume vanishing loading - i.e., $\underline{t}=0$ on $\Gamma_{N \text { nos }}, \Gamma_{N \text { nof }}$ and $\Gamma_{N}, \underline{f}=\underline{0}, \theta=0$ - for the specific purpose of analysis of energy preservation and decay. The evaluation of (28) with admissible test functions

$$
\underline{v}_{\mathrm{f}}^{*}=\underline{v}_{\mathrm{f}, h}^{n+1}, \quad \underline{v}_{\mathrm{s}}^{*}=\underline{v}_{\mathrm{s}, h}^{n+\frac{1}{2}}, \quad \underline{u}_{\mathrm{s}}^{*}=\underline{u}_{\mathrm{s}, h}^{n+\frac{1}{2}}, \quad \text { and } \quad p^{*}=p_{h}^{n+1}
$$

gives

$$
\begin{aligned}
& 0=\rho_{\mathrm{f}}\left(D_{\tau} \underline{v}_{\mathrm{f}, h}^{n+\frac{1}{2}}, \underline{v}_{\mathrm{f}, h}^{n+1}\right)_{\bar{\phi}, \Omega}+\frac{1}{\kappa_{\mathrm{S}}}\left(D_{\tau} p_{h}^{n+\frac{1}{2}}, p_{h}^{n+1}\right)_{(1-\bar{\phi})^{2}, \Omega}+\left(D_{\tau} \underline{u}_{\mathrm{s}, h}^{n+\frac{1}{2}}, \underline{u}_{\mathrm{s}, h}^{n+\frac{1}{2}}\right)_{\text {skel }} \\
& +\rho_{\mathrm{s}}\left(D_{\tau} \underline{v}_{\mathrm{s}, h}^{n+\frac{1}{2}}, \underline{v}_{\mathrm{s}, h}^{n+\frac{1}{2}}\right)_{1-\bar{\phi}, \Omega}+2\left\|\underline{\underline{\varepsilon}}\left(\underline{v}_{\mathrm{f}, h}^{n+1}\right)\right\|_{\mu_{\mathrm{f}} \bar{\phi}, \Omega}^{2}+2\left\|\underline{\underline{\varepsilon}}\left(\underline{v}_{\mathrm{s}, h}^{n+\frac{1}{2}}\right)\right\|_{\mu_{\mathrm{s}, \Omega}}^{2}+\left\|\underline{v}_{\mathrm{f}, h}^{n+1}-\underline{v}_{\mathrm{s}, h}^{n+\frac{1}{2}}\right\|_{\bar{\phi}^{2} \underline{\underline{k}}_{\mathrm{f}}^{-1}, \Omega}^{2} \\
& +\frac{\gamma \mu_{\mathrm{f}}}{h}\left\|\underline{v}_{\mathrm{f}, h}^{n+1}-\underline{v}_{\mathrm{s}, h}^{n+\frac{1}{2}}\right\|_{\bar{\phi}, \Gamma_{N \mathrm{nos}}}^{2}+\frac{\gamma \mu_{\mathrm{f}}}{h}\left\|\left(\underline{v}_{\mathrm{f}, h}^{n+1}-\underline{v}_{\mathrm{s}, h}^{n+\frac{1}{2}}\right) \cdot \underline{n}\right\|_{\bar{\phi}, \Gamma_{N \text { nof }}}^{2} \\
& -4 \mu_{\mathrm{f}}\left(\underline{\underline{\varepsilon}}\left(\underline{v}_{\mathrm{f}, h}^{n+1}\right) \cdot \underline{n}, \underline{v}_{\mathrm{f}, h}^{n+1}-\underline{v}_{\mathrm{s}, h}^{n+\frac{1}{2}}\right)_{\bar{\phi}, \Gamma_{N \mathrm{nos}}}-4 \mu_{\mathrm{f}}\left(\left(\underline{\underline{\varepsilon}}\left(\underline{v}_{\mathrm{f}, h}^{n+1}\right) \cdot \underline{n}\right) \cdot \underline{n},\left(\underline{v}_{\mathrm{f}, h}^{n+1}-\underline{v}_{\mathrm{s}, h}^{n+\frac{1}{2}}\right) \cdot \underline{n}\right)_{\bar{\phi}, \Gamma_{N \mathrm{nof}}} \text {. }
\end{aligned}
$$


We recognize here very similar terms to those in the semi-discrete identity (27), and we therefore use the same inverse inequality technique to infer, under the exact same assumptions,

$$
\begin{aligned}
\rho_{\mathrm{f}}\left(D_{\tau} \underline{v}_{\mathrm{f}, h}^{n+\frac{1}{2}}, \underline{v}_{\mathrm{f}, h}^{n+1}\right)_{\bar{\phi}, \Omega}+\frac{1}{\kappa_{\mathrm{s}}}\left(D_{\tau} p_{h}^{n+\frac{1}{2}}, p_{h}^{n+1}\right)_{(1-\bar{\phi})^{2}, \Omega}+\left(D_{\tau} \underline{u}_{\mathrm{s}, h}^{n+\frac{1}{2}}, \underline{u}_{\mathrm{s}, h}^{n+\frac{1}{2}}\right)_{\mathrm{skel}} \\
+\rho_{\mathrm{s}}\left(D_{\tau} \underline{v}_{\mathrm{s}, h}^{n+\frac{1}{2}}, \underline{v}_{\mathrm{s}, h}^{n+\frac{1}{2}}\right)_{1-\bar{\phi}, \Omega}+2\left\|\underline{\underline{\varepsilon}}\left(\underline{v}_{\mathrm{s}, h}^{n+\frac{1}{2}}\right)\right\|_{\mu_{\mathrm{s}}, \Omega}^{2}+\left\|\underline{v}_{\mathrm{f}, h}^{n+1}-\underline{v}_{\mathrm{s}, h}^{n+\frac{1}{2}}\right\|_{\bar{\phi}^{2} \underline{\underline{f}}_{\mathrm{f}}^{-1}, \Omega}^{2} \leq 0 .
\end{aligned}
$$

Defining $\mathcal{E}_{h}^{n}=\left\|X_{h}^{n}\right\|_{\mathcal{E}}^{2}$ and applying twice the identity $\langle a-b, a\rangle=\frac{1}{2}\|a\|^{2}-\frac{1}{2}\|b\|^{2}+\frac{1}{2}\|a-b\|^{2}$ to $\underline{v}_{\mathrm{f}}$ and to $p$, we get

$$
\begin{aligned}
\frac{\mathcal{E}_{h}^{n+1}-\mathcal{E}_{h}^{n}}{\Delta t}+\frac{\rho_{\mathrm{f}}}{2 \Delta t}\left\|\underline{v}_{\mathrm{f}, h}^{n+1}-\underline{v}_{\mathrm{f}, h}^{n}\right\|_{\bar{\phi}, \Omega}^{2}+\frac{1}{2 \kappa_{\mathrm{s}} \Delta t}\left\|p_{h}^{n+1}-p_{h}^{n}\right\|_{(1-\bar{\phi})^{2}, \Omega}^{2} \\
+2\left\|\underline{\underline{\varepsilon}}\left(\underline{v}_{\mathrm{s}, h}^{n+\frac{1}{2}}\right)\right\|_{\mu_{\mathrm{s}}, \Omega}^{2}+\left\|\underline{v}_{\mathrm{f}, h}^{n+1}-\underline{v}_{\mathrm{s}, h}^{n+\frac{1}{2}}\right\|_{\bar{\phi}^{2} \underline{k}_{\mathrm{f}}^{-1}, \Omega}^{2} \leq 0,
\end{aligned}
$$

hence, the discrete energy stability is guaranteed, with additional numerical dissipation terms due to backward-Euler time discretization for $\underline{v}_{\mathrm{f}}$ and $p$.

\subsection{Inf-sup stability property}

We introduce the following discrete norm, associated with the stabilisation strategy,

$$
\begin{aligned}
\|X\|_{h}^{2} \hat{=}\left\|\underline{v}_{\mathrm{f}}\right\|_{H^{1}(\Omega)}^{2}+\left\|\underline{v}_{\mathrm{s}}\right\|_{H^{1}(\Omega)}^{2}+\left\|\underline{u}_{\mathrm{s}}\right\|_{H^{1}(\Omega)}^{2}+\|p\|_{L^{2}(\Omega)}^{2} & \\
& +\frac{\gamma \mu}{h}\left\|\underline{v}_{\mathrm{f}}-\underline{v}_{\mathrm{s}}\right\|_{L^{2}\left(\Gamma_{N \mathrm{nos}}\right)}^{2}+\frac{\gamma \mu}{h}\left\|\left(\underline{v}_{\mathrm{f}}-\underline{v}_{\mathrm{s}}\right) \cdot \underline{n}\right\|_{L^{2}\left(\Gamma_{N \mathrm{nof}}\right)}^{2}+\left\|\underline{v}_{\mathrm{f}}-\underline{v}_{\mathrm{s}}\right\|_{L^{2}(\Omega)}^{2} .
\end{aligned}
$$

In addition to the assumption (18) already introduced for $\bar{\phi}$, we also assume

$$
\exists\left(\alpha_{1}, \alpha_{2}\right) \in\left(\mathbb{R}^{+}\right)^{2} \mid \forall(\underline{\mathrm{x}}, \underline{\omega}) \in \Omega \times \mathbb{R}^{3}, \alpha_{1} \underline{\omega}^{2} \leq \underline{\omega} \cdot \underline{\underline{k}}_{\mathrm{f}}(\underline{\mathrm{x}}) \cdot \underline{\omega} \leq \alpha_{2} \underline{\omega}^{2},
$$

in order to ensure the equivalence between the norms $\|\cdot\|_{\bar{\phi}, \Omega},\|\cdot\|_{(1-\bar{\phi}), \Omega},\|\cdot\|_{(1-\bar{\phi})^{2}, \Omega},\|\cdot\|_{\bar{\phi}^{2} \underline{k}_{f}^{-1}, \Omega}$, $\|\cdot\|_{L^{2}(\Omega)}$.

Our objective in this section is to establish an inf-sup stability property for $M_{h}$ defined by (25) in the norm $\|\cdot\|_{h}$.

We start with a preliminary result that we will need in the following proof to construct adequate fluid and solid test velocity fields. Let $\mathcal{V}_{h}$ and $\mathcal{Q}_{h}$ be finite dimensional subspaces of Hilbert spaces $\mathcal{V}$ and $\mathcal{Q}$ with scalar products $(\cdot, \cdot)_{\mathcal{V}}$ and $(\cdot, \cdot)_{\mathcal{Q}}$. Let $a(\cdot, \cdot)$ and $b(\cdot, \cdot)$ be continuous linear forms on $\mathcal{V} \times \mathcal{V}$ and $\mathcal{V} \times \mathcal{Q}$, and $\ell$ and $g$ linear forms on $\mathcal{V}$ and $\mathcal{Q}$. We look for $\left(v_{h}, p_{h}\right)$ in $\mathcal{V}_{h} \times \mathcal{Q}_{h}$ solution of

$$
\left\{\begin{aligned}
a\left(v_{h}, v^{*}\right)+b\left(v^{*}, p_{h}\right) & =\ell\left(v^{*}\right), & & \forall v^{*} \in \mathcal{V}_{h}, \\
b\left(v_{h}, p^{*}\right) & =g\left(p^{*}\right), & & \forall p^{*} \in \mathcal{Q}_{h} .
\end{aligned}\right.
$$

We can introduce operators $A_{h}$ from $\mathcal{V}_{h}$ to $\mathcal{V}_{h}^{\prime}$ and $B_{h}$ from $\mathcal{V}_{h}$ to $\mathcal{Q}_{h}^{\prime}$. B denotes the continuous operator from $\mathcal{V}$ to $\mathcal{Q}^{\prime}$.

\section{Lemma 3}

Let us suppose that $\operatorname{Ker} B^{t}=\{0\}$, and that $a(\cdot, \cdot)$ is coercive on $\mathcal{V}$, i.e., there exists $\alpha_{0}>0$ such that

$$
a(v, v) \geq \alpha_{0}\|v\|_{\mathcal{V}}^{2}, \quad \forall v \in \mathcal{V} .
$$


If, moreover, $b$ satisfies an inf-sup property, that is, there exists $k_{0}>0$ independent of $h$ such that

$$
\inf _{p \in \mathcal{Q}_{h v \in \mathcal{V}_{h}}} \sup _{\|v\|_{\mathcal{V}}\|p\|_{\mathcal{Q}}} \geq k_{0},
$$

then, the system (31) has a unique solution $\left(u_{h}, p_{h}\right)$. In addition, one has the bound

$$
\left\{\begin{array}{l}
\left\|v_{h}\right\|_{\mathcal{V}} \leq \frac{1}{\alpha_{0}}\|\ell\|_{\mathcal{V}^{\prime}}+\left(\frac{\|a\|}{\alpha_{0}}+1\right) \frac{1}{k_{0}}\|g\|_{\mathcal{Q}^{\prime}}, \\
\left\|p_{h}\right\|_{\mathcal{Q}} \leq \frac{1}{k_{0}}\left(\frac{\|a\|}{\alpha_{0}}+1\right)\|\ell\|_{\mathcal{V}^{\prime}}+\frac{\|a\|}{k_{0}^{2}}\left(\frac{\|a\|}{\alpha_{0}}+1\right)\|g\|_{\mathcal{Q}^{\prime}}
\end{array}\right.
$$

Proof. The inf-sup condition (32) ensures that $\operatorname{Ker} B_{h}^{t}=\{0\}$, then [2, Prop. 2.2, §II.2] ensures that $g \in \operatorname{Im} B_{h}$. The ellipticity of $a$ on $\mathcal{V}_{h}$ inherits from the one on $\mathcal{V}$, and the application of $[2$, Thm 1.1, §II.1] on the discrete problem gives the result.

\section{Remark 1}

In the case of a Stokes problem, $a(\cdot, \cdot)$ is elliptic and it is known that $\operatorname{Ker} B^{t}=\operatorname{Ker}(-\operatorname{grad})=\{0\}$ when a Dirichlet condition is applied on a non-empty portion of the boundary, so the issue is in checking (32), with $b(v, q)=\int_{\Omega} q \operatorname{div} v d \Omega$.

\section{Proposition 4 (Inf-sup stability for $M_{h}$ )}

Let us make the following assumptions:

- there exists $\lambda_{\mathrm{f}}$ such that

$$
\inf _{p_{h} \in \mathcal{Q}_{h}} \sup _{\underline{v}_{f, h} \in \mathcal{W}_{h}^{f}} \frac{\left(p, \operatorname{div}\left(\bar{\phi} \underline{v}_{f, h}\right)\right)_{\Omega}}{\left\|p_{h}\right\|_{L^{2}(\Omega)}\left\|\underline{v}_{\mathrm{f}, h}\right\|_{H^{1}(\Omega)}} \geq \lambda_{\mathrm{f}}>0,
$$

- there exists $\lambda_{\mathrm{s}}$ such that

$$
\inf _{p_{h} \in \mathcal{Q}_{h}} \sup _{\underline{v}_{\mathbf{s}, h} \in \mathcal{W}_{h}^{s}} \frac{\left(p_{h}, \operatorname{div}\left((1-\bar{\phi}) \underline{v}_{\mathrm{s}, h}\right)\right)_{\Omega}}{\left\|p_{h}\right\|_{L^{2}(\Omega)}\left\|\underline{v}_{\mathrm{s}, h}\right\|_{H^{1}(\Omega)}} \geq \lambda_{\mathrm{s}}>0,
$$

- $\mathcal{W}_{h}^{f}$ and $\mathcal{W}_{h}^{s}$ have identical traces on $\Gamma_{N \text { nos }} \cup \Gamma_{N \text { nof }}$, that is

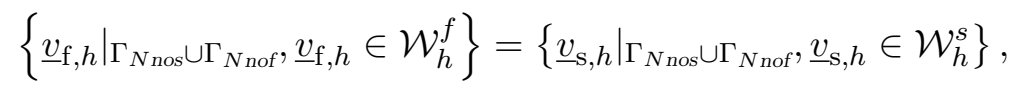

- the Robin coefficient satisfies

$$
\gamma>2 C_{i e}
$$

Then the operator $M_{h}$ defined by (25) satisfies an inf-sup property for $\|\cdot\|_{h}$, i.e. there exists $\lambda>0$ independent of $h$ such that

$$
\inf _{X_{h} \in \mathcal{V}_{h}} \sup _{X_{h}^{*} \in \mathcal{V}_{h}} \frac{M_{h}\left(X_{h}, X_{h}^{*}\right)}{\left\|X_{h}\right\|_{h}\left\|X_{h}^{*}\right\|_{h}} \geq \lambda
$$

or equivalently

$$
\forall X_{h} \in \mathcal{V}_{h}, \exists X_{h}^{*} \in \mathcal{V}_{h} \text { such that }\left\|X_{h}^{*}\right\|_{h} \leq\left\|X_{h}\right\|_{h} \text { and } M_{h}\left(X_{h}, X_{h}^{*}\right) \geq \lambda\left\|X_{h}\right\|_{h}^{2} .
$$




\section{Remark 2}

When $\bar{\phi}$ is homogeneous in space, the condition (34) takes the following form

$$
\exists \lambda_{\mathrm{f}}>0 \mid \inf _{p_{h} \in \mathcal{Q}_{h}} \sup _{\underline{v}_{\mathrm{f}, h} \in \mathcal{W}_{h}^{f}} \frac{\left(p_{h}, \operatorname{div} \underline{v}_{\mathrm{f}, h}\right)_{\Omega}}{\left\|p_{h}\right\|_{L^{2}(\Omega)}\left\|\underline{v}_{\mathrm{f}, h}\right\|_{H^{1}(\Omega)}} \geq \lambda_{\mathrm{f}}>0 .
$$

In the numerical procedure, the condition (40) is easier to satisfy than (34) because it does not involve $\bar{\phi}$, and in fact reduces to the usual Stokes inf-sup condition on $\mathcal{W}_{h}^{f} \times \mathcal{Q}_{h}$ for variables $\underline{v}_{f, h}$ and $p_{h}$.

Proof. For any $X_{h}=\left(\underline{u}_{\mathrm{s}, h}, \underline{v}_{\mathrm{s}, h}, \underline{v}_{\mathrm{f}, h}, p_{h}\right)$ in $\mathcal{V}_{h}$, we will build $X_{h}^{*} \in \mathcal{V}_{h}$ that satisfies (39) in several steps, in order to control the different terms that appear in $\|\cdot\|_{h}$.

(i) We choose $X_{h}^{1}=\left(-\underline{v}_{\mathrm{s}, h}, 0,0,0\right)$. Then

$$
M_{h}\left(X_{h}, X_{h}^{1}\right) \geq \alpha_{1}\left\|\underline{v}_{\mathrm{s}, h}\right\|_{H^{1}(\Omega)}^{2},
$$

with $\alpha_{1}>0$, due to the Korn inequality.

(ii) We pick $X_{h}^{2}=X_{h}$. The bound already derived for the energy estimate of Section 2.4 gives, for any $L>0$,

$$
\begin{aligned}
M_{h}\left(X_{h}, X_{h}^{2}\right) \geq 2 \mu_{\mathrm{f}}\left(1-\frac{C_{i e} L}{h}\right)\left\|\underline{\underline{\varepsilon}}\left(\underline{v}_{\mathrm{f}, h}\right)\right\|_{\bar{\phi}, \Omega}^{2}+2\left\|\underline{\underline{\varepsilon}}\left(\underline{v}_{\mathrm{s}, h}\right)\right\|_{\mu_{\mathrm{s}}, \Omega}^{2} \\
+\mu_{\mathrm{f}}\left(\frac{\gamma}{h}-\frac{2}{L}\right)\left\|\underline{v}_{\mathrm{f}, h}-\underline{v}_{\mathrm{s}, h}\right\|_{\bar{\phi}, \Gamma_{N \mathrm{nos}}}^{2}+\mu_{\mathrm{f}}\left(\frac{\gamma}{h}-\frac{2}{L}\right)\left\|\left(\underline{v}_{\mathrm{f}, h}-\underline{v}_{\mathrm{s}, h}\right) \cdot \underline{n}\right\|_{\bar{\phi}, \Gamma_{N \mathrm{nof}}}^{2} \\
+\left\|\underline{v}_{\mathrm{f}, h}-\underline{v}_{\mathrm{s}, h}\right\|_{\bar{\phi}^{2} \underline{k}_{\mathrm{f}}^{-1}, \Omega}^{2} .
\end{aligned}
$$

Thanks to the assumption on $\gamma$ and norm equivalences, we can obtain $\alpha_{2}>0$ such that

$$
\begin{aligned}
M_{h}\left(X_{h}, X_{h}^{2}\right) \geq \alpha_{2}\left(\left\|\underline{v}_{\mathrm{f}, h}\right\|_{H^{1}(\Omega)}^{2}+\right. & \frac{\gamma \mu_{\mathrm{f}}}{h}\left\|\underline{v}_{\mathrm{f}, h}-\underline{v}_{\mathrm{s}, h}\right\|_{L^{2}\left(\Gamma_{N \mathrm{nos}}\right)}^{2} \\
& \left.+\frac{\gamma \mu_{\mathrm{f}}}{h}\left\|\left(\underline{v}_{\mathrm{f}, h}-\underline{v}_{\mathrm{s}, h}\right) \cdot \underline{n}_{L^{2}\left(\Gamma_{N \mathrm{nof}}\right)}^{2}+\right\| \underline{v}_{\mathrm{f}, h}-\underline{v}_{\mathrm{s}, h} \|_{L^{2}(\Omega)}^{2}\right) .
\end{aligned}
$$

(iii) Thanks to (36), we define $\mathcal{L}\left(\underline{u}_{\mathrm{s}, h}\right)$ a lifting of $\left.\underline{u}_{\mathrm{s}, h}\right|_{\Gamma_{N \text { nos }} \cup \Gamma_{N \text { nof }}}$ in $\mathcal{W}_{h}^{f}$ satisfying

$$
\left\|\mathcal{L}\left(\underline{u}_{\mathrm{s}, h}\right)\right\|_{H^{1}(\Omega)} \leq C\left\|\underline{u}_{\mathrm{s}, h}\right\|_{H^{1}(\Omega)} .
$$

Given the inf-sup condition (34), Lemma 3 ensures the existence of $\left(\underline{w}_{h}^{3}, p_{h}^{3}\right)$ in $\mathcal{W}_{h}^{f} \times \mathcal{Q}_{h}$ such that for any $\left(\underline{w}_{h}^{*}, p_{h}^{*}\right)$ in $\mathcal{W}_{h}^{f} \times \mathcal{Q}_{h}$

$$
\left\{\begin{aligned}
\left(\underline{\underline{\varepsilon}}\left(\underline{w}_{h}^{3}\right), \underline{\underline{\varepsilon}}\left(\underline{w}_{h}^{*}\right)\right)_{\Omega}+\left(p_{h}^{3}, \operatorname{div}\left(\bar{\phi} \underline{w}_{h}^{*}\right)\right)_{\Omega} & =0, \\
\left(p_{h}^{*}, \operatorname{div}\left(\bar{\phi} \underline{w}_{h}^{3}\right)\right)_{\Omega} & =-\left(p_{h}^{*}, \operatorname{div}\left[(1-\bar{\phi}) \underline{u}_{\mathrm{s}, h}+\bar{\phi} \mathcal{L}\left(\underline{u}_{\mathrm{s}, h}\right)\right]\right)_{\Omega}, \\
\left.\underline{w}_{h}^{3}\right|_{\Gamma_{N \mathrm{nos}} \cup \Gamma_{N \mathrm{nof}}} & =0,
\end{aligned}\right.
$$

and from (33)

$$
\left\|\underline{w}_{h}^{3}\right\|_{H^{1}(\Omega)} \leq C\left(\left\|\underline{u}_{\mathrm{s}, h}\right\|_{H^{1}(\Omega)}+\left\|\mathcal{L}\left(\underline{u}_{\mathrm{s}, h}\right)\right\|_{H^{1}(\Omega)}\right) \leq C\left\|\underline{u}_{\mathrm{s}, h}\right\|_{H^{1}(\Omega)} .
$$


Now, let us construct $X_{h}^{3}=\left(0, \underline{u}_{\mathrm{s}, h}, \underline{v}_{\mathrm{f}, h}^{3}, 0\right)$, with $\underline{v}_{\mathrm{f}, h}^{3}=\mathcal{L}\left(\underline{u}_{\mathrm{s}, h}\right)+\underline{w}_{h}^{3}$. We then have

$$
\left\|\underline{v}_{\mathrm{f}, h}^{3}\right\|_{H^{1}(\Omega)} \leq C\left\|\underline{u}_{\mathrm{s}, h}\right\|_{H^{1}(\Omega)} .
$$

The purpose of this construction is to get $\underline{v}_{\mathrm{f}, h}^{3}$ such that $\left(p, \operatorname{div}\left(\bar{\phi} \underline{v}_{\mathrm{f}, h}^{3}+(1-\bar{\phi}) \underline{u}_{\mathrm{s}, h}\right)\right)_{\Omega}=0$ and $\underline{v}_{\mathrm{f}, h}^{3}=\underline{u}_{\mathrm{s}, h}$ on $\Gamma_{N \text { nos }} \cup \Gamma_{N \text { nof }}$, which finally allows to write that, for any $\left(\eta_{1}, \eta_{2}, \eta_{3}\right)$ in $\left(\mathbb{R}^{+}\right)^{3}$

$$
\begin{aligned}
& M_{h}\left(X_{h}, X_{h}^{3}\right)=2 \mu_{\mathrm{f}}\left(\underline{\underline{\varepsilon}}\left(\underline{v}_{\mathrm{f}, h}\right), \underline{\underline{\varepsilon}}\left(\underline{v}_{\mathrm{f}, h}^{3}\right)\right)_{\bar{\phi}, \Omega}+\left(\underline{u}_{\mathrm{s}, h}, \underline{u}_{\mathrm{s}, h}\right)_{\mathrm{skel}}+2\left(\underline{\underline{\varepsilon}}\left(\underline{v}_{\mathrm{s}, h}\right), \underline{\underline{\varepsilon}}\left(\underline{u}_{\mathrm{s}, h}\right)\right)_{\mu_{\mathrm{s}}, \Omega} \\
&-2 \mu_{\mathrm{f}}\left(\underline{\underline{\varepsilon}}\left(\underline{v}_{\mathrm{f}, h}^{3}\right) \cdot \underline{n}, \underline{v}_{\mathrm{f}, h}-\underline{v}_{\mathrm{s}, h}\right)_{\bar{\phi}, \Gamma_{N \mathrm{nos}}}-2 \mu_{\mathrm{f}}\left(\left(\underline{\underline{\varepsilon}}\left(\underline{v}_{\mathrm{f}, h}^{3}\right) \cdot \underline{n}\right) \cdot \underline{n},\left(\underline{v}_{\mathrm{f}, h}-\underline{v}_{\mathrm{s}, h}\right) \cdot \underline{n}\right)_{\bar{\phi}, \Gamma_{N \mathrm{nof}}} \\
&+\left(\underline{v}_{\mathrm{f}, h}-\underline{v}_{\mathrm{s}, h}, \underline{v}_{\mathrm{f}, h}^{3}-\underline{u}_{\mathrm{s}, h}\right)_{\bar{\phi}^{2} \underline{k}_{\mathrm{f}}^{-1}, \Omega} .
\end{aligned}
$$

Using Young's inequality we have

$$
\begin{aligned}
& M_{h}\left(X_{h}, X_{h}^{3}\right) \geq \alpha\left\|\underline{u}_{\mathrm{s}, h}\right\|_{H^{1}(\Omega)}^{2} \\
& -\mu_{\mathrm{f}}\left(\eta_{1}\left\|\underline{\underline{\varepsilon}}\left(\underline{v}_{\mathrm{f}, h}^{3}\right)\right\|_{\bar{\phi}, \Omega}^{2}+\frac{1}{\eta_{1}}\left\|\underline{\underline{\varepsilon}}\left(\underline{v}_{\mathrm{f}, h}\right)\right\|_{\bar{\phi}, \Omega}^{2}\right)-\mu_{\mathrm{f}}\left(\eta_{2} h\left\|\underline{\underline{\varepsilon}}\left(\underline{v}_{\mathrm{f}, h}^{3}\right)\right\|_{\bar{\phi}, \Gamma_{N \mathrm{nos}} \cup \Gamma_{N \mathrm{nof}}}^{2}\right. \\
& \left.+\frac{1}{\eta_{2} h}\left\|\underline{v}_{\mathrm{f}, h}-\underline{v}_{\mathrm{s}, h}\right\|_{\bar{\phi}, \Gamma_{N \mathrm{nos}}}^{2}+\frac{1}{\eta_{2} h}\left\|\left(\underline{v}_{\mathrm{f}, h}-\underline{v}_{\mathrm{s}, h}\right) \cdot \underline{n}\right\|_{\bar{\phi}, \Gamma_{N \mathrm{nof}}}^{2}\right) \\
& -\left(\frac{1}{2 \eta_{3}}\left\|\underline{v}_{\mathrm{f}, h}-\underline{v}_{\mathrm{s}, h}\right\|_{\bar{\phi}^{2} \underline{k}_{\mathrm{f}}^{-1}, \Omega}^{2}+\frac{\eta_{3}}{2}\left\|\underline{v}_{\mathrm{f}, h}^{3}-\underline{u}_{\mathrm{s}, h}\right\|_{\bar{\phi}^{2} \underline{\underline{f}}_{\mathrm{f}}^{-1}, \Omega}^{2}\right) \\
& -\left(\frac{1}{\eta_{4}}\left\|\underline{\underline{\varepsilon}}\left(\underline{v}_{\mathrm{s}, h}\right)\right\|_{\mu_{\mathrm{s}}, \Omega}^{2}+\eta_{4}\left\|\underline{\underline{\varepsilon}}\left(\underline{u}_{\mathrm{s}, h}\right)\right\|_{\mu_{\mathrm{s}}, \Omega}^{2}\right)
\end{aligned}
$$

We can now employ the inverse inequality (26) and various other standard bounds to infer

$$
\begin{array}{r}
M_{h}\left(X_{h}, X_{h}^{3}\right) \geq\left[\alpha-C\left(\eta_{1}+\eta_{2}+\eta_{3}+\eta_{4}\right)\right]\left\|\underline{u}_{\mathrm{s}, h}\right\|_{H^{1}(\Omega)}^{2} \\
-C^{\prime}\left(\frac{1}{\eta_{1}}\left\|\underline{v}_{\mathrm{f}, h}\right\|_{H^{1}(\Omega)}^{2}+\frac{1}{\eta_{2}} \frac{\gamma \mu_{\mathrm{f}}}{h}\left\|\underline{v}_{\mathrm{f}, h}-\underline{v}_{\mathrm{s}, h}\right\|_{L^{2}\left(\Gamma_{N \mathrm{nos}}\right)}^{2}+\frac{1}{\eta_{2}} \frac{\gamma \mu_{\mathrm{f}}}{h} \|\left(\underline{v}_{\mathrm{f}, h}-\underline{v}_{\mathrm{s}, h}\right) \cdot \underline{n}_{L^{2}\left(\Gamma_{N \mathrm{nof}}\right)}^{2}\right. \\
\left.\quad+\frac{1}{\eta_{3}}\left\|\underline{v}_{\mathrm{f}, h}-\underline{v}_{\mathrm{s}, h}\right\|_{L^{2}(\Omega)}^{2}+\frac{1}{\eta_{4}}\left\|\underline{v}_{\mathrm{s}, h}\right\|_{H^{1}(\Omega)}^{2}\right),
\end{array}
$$

and we see that we can easily pick our constants $\left(\eta_{1}, \eta_{2}, \eta_{3}, \eta_{4}\right)$ to obtain $\alpha_{3}$ and $\beta_{3}$ strictly positive such that

$$
\begin{aligned}
M_{h}\left(X_{h}, X_{h}^{3}\right) \geq \alpha_{3}\left\|\underline{u}_{\mathrm{s}, h}\right\|_{H^{1}(\Omega)}^{2}-\beta_{3}\left(\left\|\underline{v}_{\mathrm{f}, h}\right\|_{H^{1}(\Omega)}^{2}+\frac{\gamma \mu_{\mathrm{f}}}{h}\left\|\underline{v}_{\mathrm{f}, h}-\underline{v}_{\mathrm{s}, h}\right\|_{L^{2}\left(\Gamma_{N \mathrm{nos}}\right)}^{2}\right. \\
\left.\quad+\frac{\gamma \mu_{\mathrm{f}}}{h}\left\|\left(\underline{v}_{\mathrm{f}, h}-\underline{v}_{\mathrm{s}, h}\right) \cdot \underline{n}\right\|_{L^{2}\left(\Gamma_{N \mathrm{nof}}\right)}^{2}+\left\|\underline{v}_{\mathrm{f}, h}-\underline{v}_{\mathrm{s}, h}\right\|_{L^{2}(\Omega)}^{2}+\left\|\underline{v}_{\mathrm{s}, h}\right\|_{H^{1}(\Omega)}^{2}\right) .
\end{aligned}
$$

In addition, there exists $\gamma_{3}>0$ such that

$$
\begin{aligned}
& \left\|X_{h}^{3}\right\|_{h}^{2}=\left\|\underline{v}_{\mathrm{f}, h}^{3}\right\|_{H^{1}(\Omega)}^{2}+\left\|\underline{u}_{\mathrm{s}, h}\right\|_{H^{1}(\Omega)}^{2}+\left\|\underline{v}_{\mathrm{f}, h}^{3}-\underline{u}_{\mathrm{s}, h}\right\|_{L^{2}(\Omega)}^{2} \\
& \quad \leq\left\|\underline{v}_{\mathrm{f}, h}^{3}\right\|_{H^{1}(\Omega)}^{2}+\left\|\underline{u}_{\mathrm{s}, h}\right\|_{H^{1}(\Omega)}^{2}+2\left\|\underline{v}_{\mathrm{f}, h}^{3}\right\|_{L^{2}(\Omega)}^{2}+2\left\|\underline{u}_{\mathrm{s}, h}\right\|_{L^{2}(\Omega)}^{2} \leq \gamma_{3}\left\|\underline{u}_{\mathrm{s}, h}\right\|_{H^{1}(\Omega)}^{2} \leq \gamma_{3}\left\|X_{h}\right\|_{h} .
\end{aligned}
$$


(iv) As a consequence of (34), we can construct $\underline{v}_{\mathrm{f}, h}^{4}$ such that

$$
\left\{\begin{array}{c}
\left\|\underline{v}_{\mathrm{f}, h}^{4}\right\|_{H^{1}(\Omega)} \leq\left\|p_{h}\right\|_{L^{2}(\Omega)}, \\
-\left(p_{h}, \operatorname{div}\left(\bar{\phi} \underline{v}_{\mathrm{f}, h}^{4}\right)\right)_{\Omega} \geq \lambda_{\mathrm{f}}\left\|p_{h}\right\|_{L^{2}(\Omega)}^{2}
\end{array}\right.
$$

Once again, (36) allows to introduce $\mathcal{L}\left(\underline{v}_{\mathrm{f}, h}^{4}\right)$ a lifting of $\underline{v}_{\mathrm{f}, h}^{4} \mid \Gamma_{N_{\mathrm{nos}}} \cup \Gamma_{N_{\mathrm{nof}}}$ in $\mathcal{W}_{h}^{s}$ such that

$$
\left\|\mathcal{L}\left(\underline{v}_{\mathrm{f}, h}^{4}\right)\right\|_{H^{1}(\Omega)} \leq C\left\|\underline{v}_{\mathrm{f}, h}^{4}\right\|_{H^{1}(\Omega)} .
$$

Thanks to (35), Lemma 3 ensures the existence of $\left(\underline{w}_{h}^{4}, p_{h}^{4}\right)$ in $\mathcal{W}_{h}^{s} \times \mathcal{Q}_{h}$ such that for any $\left(\underline{w}^{*}, p^{*}\right)$ in $\mathcal{W}_{h}^{s} \times \mathcal{Q}_{h}$,

$$
\left\{\begin{aligned}
\left(\underline{\underline{\varepsilon}}\left(\underline{w}_{h}^{4}\right), \underline{\underline{\varepsilon}}\left(\underline{w}^{*}\right)\right)_{\Omega}+\left(p_{h}^{4}, \operatorname{div}\left((1-\bar{\phi}) \underline{w}^{*}\right)\right)_{\Omega} & =0 \\
\left(p^{*}, \operatorname{div}\left((1-\bar{\phi}) \underline{w}_{h}^{4}\right)\right)_{\Omega} & =-\left(p^{*}, \operatorname{div}\left[(1-\bar{\phi}) \mathcal{L}\left(\underline{v}_{\mathrm{f}, h}^{4}\right)\right]\right)_{\Omega}, \\
\left.\underline{w}_{h}^{4}\right|_{\Gamma_{N \mathrm{nos}} \cup \Gamma_{N \mathrm{nof}}} & =0
\end{aligned}\right.
$$

and recalling (33),

$$
\left\|\underline{w}_{h}^{4}\right\|_{H^{1}(\Omega)} \leq C\left\|\mathcal{L}\left(\underline{v}_{\mathrm{f}, h}^{4}\right)\right\|_{H^{1}(\Omega)} \leq C\left\|\underline{v}_{\mathrm{f}, h}^{4}\right\|_{H^{1}(\Omega)} .
$$

Finally, we select $X_{h}^{4}=\left(0, \underline{v}_{\mathrm{s}, h}^{4}, \underline{v}_{\mathrm{f}, h}^{4}, 0\right)$ with $\underline{v}_{\mathrm{s}, h}^{4}=\mathcal{L}\left(\underline{v}_{\mathrm{f}, h}^{4}\right)+\underline{w}_{h}^{4}$. Then

$$
\left\|\underline{v}_{\mathrm{s}, h}^{4}\right\|_{H^{1}(\Omega)} \leq\left\|\underline{w}_{h}^{4}\right\|_{H^{1}(\Omega)}+\left\|\mathcal{L}\left(\underline{v}_{\mathrm{f}, h}^{4}\right)\right\|_{H^{1}(\Omega)} \leq C\left\|\underline{v}_{\mathrm{f}, h}^{4}\right\|_{H^{1}(\Omega)} \leq C\left\|p_{h}\right\|_{L^{2}(\Omega)} .
$$

This construction provides a control on $p_{h}$ through the divergence term, neutralizing the $\underline{v}_{\mathrm{s}, h}^{4}$ contribution thanks to $\left(p^{*}, \operatorname{div}\left((1-\bar{\phi}) \underline{v}_{\mathrm{s}, h}^{4}\right)\right)_{\Omega}=0$. It also imposes $\underline{v}_{\mathrm{f}, h}^{4}=\underline{v}_{\mathrm{s}, h}^{4}$ on $\Gamma_{N \text { nos }} \cup \Gamma_{N \text { nof }}$, and finally allows to write

$$
\begin{aligned}
& M_{h}\left(X_{h}, X_{h}^{4}\right)=2 \mu_{\mathrm{f}}\left(\underline{\underline{\varepsilon}}\left(\underline{v}_{\mathrm{f}, h}\right), \underline{\underline{\varepsilon}}\left(\underline{v}_{\mathrm{f}, h}^{4}\right)\right)_{\bar{\phi}, \Omega}+\left(\underline{u}_{\mathrm{s}, h}, \underline{v}_{\mathrm{s}, h}^{4}\right)_{\mathrm{skel}}+2\left(\underline{\underline{\varepsilon}}\left(\underline{v}_{\mathrm{s}, h}\right), \underline{\underline{\varepsilon}}\left(\underline{v}_{\mathrm{s}, h}^{4}\right)\right)_{\mu_{\mathrm{s}}, \Omega} \\
&-2 \mu_{\mathrm{f}}\left(\underline{\underline{\varepsilon}}\left(\underline{v}_{\mathrm{f}, h}^{4}\right) \cdot \underline{n}, \underline{v}_{\mathrm{f}, h}-\underline{v}_{\mathrm{s}, h}\right)_{\bar{\phi}, \Gamma_{N \mathrm{nos}}}-2 \mu_{\mathrm{f}}\left(\left(\underline{\underline{\varepsilon}}\left(\underline{v}_{\mathrm{f}, h}^{4}\right) \cdot \underline{n}\right) \cdot \underline{n},\left(\underline{v}_{\mathrm{f}, h}-\underline{v}_{\mathrm{s}, h}\right) \cdot \underline{n}\right)_{\bar{\phi}, \Gamma_{N \mathrm{nof}}} \\
&+\left(\underline{v}_{\mathrm{f}, h}-\underline{v}_{\mathrm{s}, h}, \underline{v}_{\mathrm{f}, h}^{4}-\underline{v}_{\mathrm{s}, h}^{4}\right)_{\bar{\phi}^{2} \underline{\underline{k}}_{\mathrm{f}}^{-1}, \Omega}-\left(p_{h}, \operatorname{div}\left(\bar{\phi} \underline{v}_{\mathrm{f}, h}^{4}\right)\right)_{\Omega} .
\end{aligned}
$$

Young and inverse inequalities now give

$$
\begin{aligned}
M_{h}\left(X_{h}, X_{h}^{4}\right) \geq & {\left[\lambda_{\mathrm{f}}-C\left(\eta_{1}+\eta_{2}+\eta_{3}+\eta_{4}\right)\right]\left\|p_{h}\right\|_{L^{2}(\Omega)}^{2} } \\
- & C^{\prime}\left(\frac{1}{\eta_{1}}\left\|\underline{v}_{\mathrm{f}, h}\right\|_{H^{1}(\Omega)}^{2}+\frac{1}{\eta_{2}}\left\|\underline{u}_{\mathrm{s}, h}\right\|_{H^{1}(\Omega)}^{2}+\frac{1}{\eta_{3}} \frac{\gamma \mu_{\mathrm{f}}}{h}\left\|\underline{v}_{\mathrm{f}, h}-\underline{v}_{\mathrm{s}, h}\right\|_{L^{2}\left(\Gamma_{N \mathrm{nos}}\right)}^{2}\right. \\
& \left.+\frac{1}{\eta_{3}} \frac{\gamma \mu_{\mathrm{f}}}{h}\left\|\left(\underline{v}_{\mathrm{f}, h}-\underline{v}_{\mathrm{s}, h}\right) \cdot \underline{n}\right\|_{L^{2}\left(\Gamma_{N \mathrm{nof}}\right)}^{2}+\frac{1}{\eta_{4}}\left\|\underline{v}_{\mathrm{f}, h}-\underline{v}_{\mathrm{s}, h}\right\|_{L^{2}(\Omega)}^{2}+\frac{1}{\eta_{5}}\left\|\underline{v}_{\mathrm{s}, h}\right\|_{H^{1}(\Omega)}^{2}\right),
\end{aligned}
$$

and we can adjust the constants to obtain

$$
\begin{array}{r}
M_{h}\left(X_{h}, X_{h}^{4}\right) \geq \alpha_{4}\left\|p_{h}\right\|_{L^{2}(\Omega)}-\beta_{4}\left(\left\|\underline{v}_{\mathrm{f}, h}\right\|_{H^{1}(\Omega)}^{2}+\left\|\underline{u}_{\mathrm{s}, h}\right\|_{H^{1}(\Omega)}^{2}+\frac{\gamma \mu_{\mathrm{f}}}{h}\left\|\underline{v}_{\mathrm{f}, h}-\underline{v}_{\mathrm{s}, h}\right\|_{L^{2}\left(\Gamma_{N \mathrm{nos}}\right)}^{2}\right. \\
\left.+\frac{\gamma \mu_{\mathrm{f}}}{h}\left\|\left(\underline{v}_{\mathrm{f}, h}-\underline{v}_{\mathrm{s}, h}\right) \cdot \underline{n}_{L^{2}\left(\Gamma_{N \mathrm{nof}}\right)}^{2}+\right\| \underline{v}_{\mathrm{f}, h}-\underline{v}_{\mathrm{s}, h}\left\|_{L^{2}(\Omega)}^{2}+\right\| \underline{v}_{\mathrm{s}, h} \|_{H^{1}(\Omega)}^{2}\right),
\end{array}
$$

with also

$$
\left\|X_{h}^{4}\right\|_{h}^{2}=\left\|\underline{v}_{\mathrm{f}, h}^{4}\right\|_{H^{1}(\Omega)}^{2}+\left\|\underline{v}_{\mathrm{s}, h}^{4}\right\|_{H^{1}(\Omega)}^{2}+\left\|\underline{v}_{\mathrm{f}, h}^{4}-\underline{v}_{\mathrm{s}, h}^{4}\right\|_{L^{2}(\Omega)}^{2} \leq \gamma_{4}\left\|p_{h}\right\|_{L^{2}(\Omega)}^{2} \leq \gamma_{4}\left\|X_{h}\right\|_{h}^{2} .
$$


(v) Considering now $\bar{X}_{h}=X_{h}^{1}+X_{h}^{2}+\delta_{3} X_{h}^{3}+\delta_{4} X_{h}^{4}$ and gathering (41), (42), (44) and (46), we have

$$
\begin{aligned}
& M_{h}\left(X_{h}, \bar{X}_{h}\right) \geq\left(\alpha_{1}-\delta_{3} \beta_{3}-\delta_{4} \beta_{4}\right)\left\|\underline{v}_{\mathrm{s}, h}\right\|_{H^{1}(\Omega)}^{2}+\left(\alpha_{2}-\delta_{3} \beta_{3}-\delta_{4} \beta_{4}\right)\left\|\underline{v}_{\mathrm{f}, h}\right\|_{H^{1}(\Omega)}^{2} \\
& \quad+\left(\alpha_{2}-\delta_{3} \beta_{3}-\delta_{4} \beta_{4}\right)\left(\frac{\gamma \mu_{\mathrm{f}}}{h}\left\|\underline{v}_{\mathrm{f}, h}-\underline{v}_{\mathrm{s}, h}\right\|_{L^{2}\left(\Gamma_{N \mathrm{nos}}\right)}^{2}+\frac{\gamma \mu_{\mathrm{f}}}{h}\left\|\left(\underline{v}_{\mathrm{f}, h}-\underline{v}_{\mathrm{s}, h}\right) \cdot \underline{n}\right\|_{L^{2}\left(\Gamma_{N \mathrm{nof}}\right)}^{2}\right) \\
& \quad+\left(\alpha_{2}-\delta_{3} \beta_{3}-\delta_{4} \beta_{4}\right)\left\|\underline{v}_{\mathrm{f}, h}-\underline{v}_{\mathrm{s}, h}\right\|_{L^{2}(\Omega)}^{2}+\left(\delta_{3} \alpha_{3}-\delta_{4} \beta_{4}\right)\left\|\underline{u}_{\mathrm{s}, h}\right\|_{H^{1}(\Omega)}^{2}+\delta_{4} \alpha_{4}\left\|p_{h}\right\|_{L^{2}(\Omega)} .
\end{aligned}
$$

We can then first set $\delta_{3}>0$ so that $\alpha_{1}-\delta_{3} \beta_{3}>0$ and $\alpha_{2}-\delta_{3} \beta_{3}>0$, and secondly $\delta_{4}>0$ so that all coefficients remain strictly positive. This finally yields $M_{h}\left(X_{h}, \bar{X}_{h}\right) \geq \alpha\left\|X_{h}\right\|_{h}^{2}$, with $\left\|\bar{X}_{h}\right\|_{h} \leq C\left\|X_{h}\right\|_{h}$ as desired.

\section{Remark 3}

We have not used the solid viscosity to control the solid velocity in the above proof (see Step 2). Therefore, this inf-sup property holds even if $\mu_{\mathrm{s}}=0$.

\subsection{Convergence in time and space of the fully discretized scheme solution}

We introduce a new state space with more regularity $\mathcal{X}^{+}=\left(H^{2}(\Omega)^{3}\right)^{3} \times H^{1}(\Omega)$, equipped with the natural norm

$$
\|X\|_{\mathcal{X}^{+}}^{2}=\left\|\underline{u}_{\mathrm{s}}\right\|_{H^{2}(\Omega)}^{2}+\left\|\underline{v}_{\mathrm{s}}\right\|_{H^{2}(\Omega)}^{2}+\left\|\underline{v}_{\mathrm{f}}\right\|_{H^{2}(\Omega)}^{2}+\|p\|_{H^{1}(\Omega)}^{2},
$$

and we also introduce $\mathcal{X}^{-}=H^{1}(\Omega)^{3} \times L^{2}(\Omega)^{3} \times L^{2}(\Omega)^{3} \times L^{2}(\Omega)$, whose natural norm is equivalent to $\|\cdot\|_{\mathcal{E}}$.

We note that, under the assumptions of Proposition 4, existence and uniqueness of a solution $X_{h}^{n+1}$ to the discrete problem (28) follows from the invertibility of the total operator - both dynamical terms and static form $M_{h}$. This stems from the positivity of $M_{h}$ - see the proof of Proposition 4 (Step 2) - and from the following argument. At each time step, $X_{h}^{n+1}$ is the solution of a problem in which the bilinear operator to be inverted is

$$
\begin{aligned}
D_{h}\left(X_{h}^{n+1}, X_{h}^{*}\right)=\frac{\rho_{\mathrm{f}}}{\Delta t}\left(\underline{v}_{\mathrm{f}, h}^{n+1}, \underline{v}_{\mathrm{f}}^{*}\right)_{\bar{\phi}, \Omega}+\frac{\rho_{\mathrm{s}}}{\Delta t}\left(\underline{v}_{\mathrm{s}, h}^{n+1}, \underline{v}_{\mathrm{s}}^{*}\right)_{1-\bar{\phi}, \Omega}+\frac{1}{\kappa_{\mathrm{s}} \Delta t}\left(p_{h}^{n+1}, p^{*}\right)_{(1-\bar{\phi})^{2}, \Omega} \\
+\frac{1}{\Delta t}\left(\underline{u}_{\mathrm{s}, h}^{n+1}, \underline{u}_{\mathrm{s}}^{*}\right)_{\mathrm{skel}}+\frac{1}{2} M_{h}\left(\left(\underline{u}_{\mathrm{s}, h}^{n+1}, \underline{v}_{\mathrm{s}, h}^{n+1}, 2 \underline{v}_{\mathrm{f}, h}^{n+1}, 2 p_{h}^{n+1}\right),\left(\underline{u}_{\mathrm{s}}^{*}, \underline{v}_{\mathrm{s}}^{*}, \underline{v}_{\mathrm{f}}^{*}, p^{*}\right)\right) .
\end{aligned}
$$

Assuming that $X_{h}^{n+1}$ is in the kernel of this bilinear form and testing with the specific choice $X_{h}^{*}=\left(\underline{u}_{\mathrm{s}, h}^{n+1}, \underline{v}_{\mathrm{s}, h}^{n+1}, 2 \underline{v}_{\mathrm{f}, h}^{n+1}, 2 p_{h}^{n+1}\right)$, we have

$$
\begin{gathered}
0=D_{h}\left(X_{h}^{n+1}, X_{h}^{*}\right)=\frac{2 \rho_{\mathrm{f}}}{\Delta t}\left(\underline{v}_{\mathrm{f}, h}^{n+1}, \underline{v}_{\mathrm{f}, h}^{n+1}\right)_{\bar{\phi}, \Omega}+\frac{\rho_{\mathrm{s}}}{\Delta t}\left(\underline{v}_{\mathrm{s}, h}^{n+1}, \underline{v}_{\mathrm{s}, h}^{n+1}\right)_{1-\bar{\phi}, \Omega}+\frac{2}{\kappa_{\mathrm{s}} \Delta t}\left(p_{h}^{n+1}, p_{h}^{n+1}\right)_{(1-\bar{\phi})^{2}, \Omega} \\
+\frac{1}{\Delta t}\left(\underline{u}_{\mathrm{s}, h}^{n+1}, \underline{u}_{\mathrm{s}, h}^{n+1}\right)_{\mathrm{skel}}+\frac{1}{2} M_{h}\left(\left(\underline{u}_{\mathrm{s}, h}^{n+1}, \underline{v}_{\mathrm{s}, h}^{n+1}, 2 \underline{v}_{\mathrm{f}, h}^{n+1}, 2 p_{h}^{n+1}\right),\left(\underline{u}_{\mathrm{s}, h}^{n+1}, \underline{v}_{\mathrm{s}, h}^{n+1}, 2 \underline{v}_{\mathrm{f}, h}^{n+1}, 2 p_{h}^{n+1}\right)\right) \\
\geq \frac{2 \rho_{\mathrm{f}}}{\Delta t}\left\|\underline{\mathrm{f}}_{\mathrm{f}, h}^{n+1}\right\|_{\Omega, \rho_{\mathrm{f}} \bar{\phi}}^{2}+\frac{\rho_{\mathrm{s}}}{\Delta t}\left\|\underline{v}_{\mathrm{s}, h}^{n+1}\right\|_{\Omega, \rho_{\mathrm{s}}(1-\bar{\phi})}^{2}+\frac{2}{\kappa_{\mathrm{s}} \Delta t}\left\|p_{h}^{n+1}\right\|_{\Omega,(1-\bar{\phi})^{2}}^{2}+\frac{1}{\Delta t}\left\|\underline{u}_{\mathrm{s}, h}^{n+1}\right\|_{\mathrm{skel}}^{2},
\end{gathered}
$$

which necessarily implies $X_{h}^{n+1}=0$.

We are now in a position to establish the main result of this paper.

\section{Proposition 5 (Total convergence)}

Under the assumptions of Proposition 4, and assuming further that the solution $X$ of (8) has 
regularity $\mathcal{C}^{1}\left(0, T, \mathcal{X}^{+}\right) \cap \mathcal{C}^{2}\left(0, T, \mathcal{X}^{-}\right)$with $T$ the simulation time, then there exists a constant $C$ depending only on $\|X\|_{L^{\infty}\left(0, T, \mathcal{X}^{+}\right)}$, $\left\|\partial_{t} X\right\|_{L^{\infty}\left[0, T, \mathcal{X}^{+}\right]}$and $\left\|\partial_{t}^{2} X\right\|_{L^{\infty}[0, T, \mathcal{E}]}$ such that, for all $n \leq T / \Delta t$,

$$
\left\|X_{h}^{n}-X\left(t^{n}\right)\right\|_{\mathcal{E}} \leq C(h+\Delta t) .
$$

Proof. Let us introduce the projection operator $P_{h}=\left[P_{h}^{\mathrm{u}}, P_{h}^{\mathrm{s}}, P_{h}^{\mathrm{f}}, P_{h}^{\mathrm{p}}\right]$ such that,

$$
\forall X_{h}^{*}=\left(\underline{u}_{\mathrm{s}, h}^{*}, \underline{v}_{\mathrm{s}, h}^{*}, \underline{v}_{\mathrm{f}, h}^{*}, p_{h}^{*}\right) \in \mathcal{V}_{h}, \quad M_{h}\left(P_{h} X, X_{h}^{*}\right)=M_{h}\left(X, X_{h}^{*}\right) .
$$

We start with the following preliminary result.

\section{Lemma 6}

Under the assumptions of Proposition 4, for any $X^{\sharp}$ in $\mathcal{X}^{+} \cap \mathcal{V}^{0}$,

$$
\left\|P_{h} X^{\sharp}-X^{\sharp}\right\|_{\mathcal{E}} \leq C h\left\|X^{\sharp}\right\|_{\mathcal{X}^{+}} .
$$

\section{Remark 4}

More generally, with shape functions of order $k \geq 1$, we have that for any $X^{\sharp}$ in $\mathcal{X}^{k} \cap \mathcal{V}^{0}$ with $\mathcal{X}^{k}=\left(H^{k+1}(\Omega)^{3}\right)^{3} \times H^{k}(\Omega)$,

$$
\left\|P_{h} X^{\sharp}-X^{\sharp}\right\|_{\mathcal{E}} \leq C h^{k}\left\|X^{\sharp}\right\|_{\mathcal{X}^{k}},
$$

with $\|\cdot\|_{\mathcal{X}^{k}}$ the natural norm in $\mathcal{X}^{k}$. Nevertheless, for the sake of compactness and to fix the ideas, we will restrict ourselves to the lowest-order case $k=1$.

Proof of Lemma 6. Let us introduce the Clément interpolation $I_{h} X^{\sharp}$ in $\mathcal{V}_{h} \cap \mathcal{V}^{0}$ with the notation $I_{h} X^{\sharp}=\left(I_{h}^{\mathrm{u}} X^{\sharp}, I_{h}^{\mathrm{s}} X^{\sharp}, I_{h}^{\mathrm{f}} X^{\sharp}, I_{h}^{\mathrm{p}} X^{\sharp}\right)$ and that satisfies [8]

$$
\left\{\begin{aligned}
\left\|X^{\sharp}-I_{h} X^{\sharp}\right\|_{\mathcal{V}^{0}} & \leq C h\left\|X^{\sharp}\right\|_{\mathcal{X}^{+}}, \\
\left\|I_{h} X^{\sharp}\right\|_{\mathcal{V}^{0}} & \leq C\left\|X^{\sharp}\right\|_{\mathcal{V}^{0}}, \\
\left\|I_{h}^{\mathrm{p}} X^{\sharp}-p^{\sharp}\right\|_{L^{2}\left(\Gamma_{N \text { nos }} \cup \Gamma_{N \text { nof }}\right)} & \leq C h^{1 / 2}\left\|p^{\sharp}\right\|_{H^{1}(\Omega)}, \\
\left\|I_{h}^{\mathrm{f}} X^{\sharp}-\underline{v}_{\mathrm{f}}^{\sharp}\right\|_{H^{1}\left(\Gamma_{N \text { nos }} \cup \Gamma_{N \text { nof }}\right)} & \leq C h^{1 / 2}\left\|\underline{v}_{\mathrm{f}}^{\sharp}\right\|_{H^{2}(\Omega)} .
\end{aligned}\right.
$$

Proposition 4 ensures the existence of $X_{h}^{*}$ in $\mathcal{V}_{h}$ such that

$$
\left\|X_{h}^{*}\right\|_{h}=1 \text { and } M_{h}\left(P_{h} X^{\sharp}-I_{h} X^{\sharp}, X_{h}^{*}\right) \geq \alpha\left\|P_{h} X^{\sharp}-I_{h} X^{\sharp}\right\|_{h},
$$

Since, by definition of $P_{h}$, we have $M_{h}\left(P_{h} X^{\sharp}-I_{h} X^{\sharp}, X_{h}^{*}\right)=M_{h}\left(X^{\sharp}-I_{h} X^{\sharp}, X_{h}^{*}\right)$, we infer

$$
\left\|P_{h} X^{\sharp}-I_{h} X^{\sharp}\right\|_{h} \leq C M_{h}\left(X^{\sharp}-I_{h} X^{\sharp}, X_{h}^{*}\right) .
$$

Let us then look at the terms of $M_{h}\left(X^{\sharp}-I_{h} X^{\sharp}, X_{h}^{*}\right)$ that are not directly controlled by $\|\cdot\|_{h}$. Thanks to the above interpolation estimates and the Cauchy-Schwarz inequality,

$$
\begin{aligned}
\left(p^{\sharp}-I_{h}^{\mathrm{p}} X^{\sharp},\left(\underline{v}_{\mathrm{f}, h}^{*}-\underline{v}_{\mathrm{s}, h}^{*}\right) \cdot \underline{n}\right)_{\Gamma_{N \mathrm{nos}}} & \leq h^{1 / 2}\left\|p^{\sharp}-I_{h}^{\mathrm{p}} X^{\sharp}\right\|_{L^{2}\left(\Gamma_{N \mathrm{nos}}\right)} \cdot \frac{1}{h^{1 / 2}}\left\|\underline{v}_{\mathrm{f}, h}^{*}-\underline{v}_{\mathrm{s}, h}^{*}\right\|_{L^{2}\left(\Gamma_{N \mathrm{nos}}\right)} \\
& \leq C h\left\|X^{\sharp}\right\|_{\mathcal{X}^{+}}\left\|X_{h}^{*}\right\|_{h} .
\end{aligned}
$$

Similar arguments enable us to control $2 \mu_{\mathrm{f}}\left(\underline{\underline{\varepsilon}}\left(\underline{v}_{\mathrm{f}}^{\sharp}-I_{h}^{\mathrm{f}} X^{\sharp}\right) \cdot \underline{n}, \underline{v}_{\mathrm{f}, h}^{*}-\underline{v}_{\mathrm{s}, h}^{*}\right)_{\Gamma_{N \text { nol }}}$, as well as the terms associated with the boundary $\Gamma_{N \text { nof. }}$. All other terms are directly controlled with CauchySchwarz, and therefore we have

$$
M_{h}\left(X^{\sharp}-I_{h} X^{\sharp}, X_{h}^{*}\right) \leq C h\left\|X^{\sharp}\right\|_{\mathcal{X}^{+}}\left\|X_{h}^{*}\right\|_{h},
$$


which, together with (49), gives

$$
\left\|P_{h} X^{\sharp}-I_{h} X^{\sharp}\right\|_{h} \leq C h\left\|X^{\sharp}\right\|_{\mathcal{X}^{+}} \text {. }
$$

Finally, using $\|\cdot\|_{\mathcal{E}} \leq\|\cdot\|_{h}$, the triangular inequality gives

$$
\begin{aligned}
\left\|P_{h} X^{\sharp}-X^{\sharp}\right\|_{\mathcal{E}} & \leq\left\|P_{h} X^{\sharp}-I_{h} X^{\sharp}\right\|_{\mathcal{E}}+\left\|I_{h} X^{\sharp}-X^{\sharp}\right\|_{\mathcal{E}} \\
& \leq C h\left\|X^{\sharp}\right\|_{\mathcal{X}^{+}}+C h\left\|X^{\sharp}\right\|_{\mathcal{X}^{+}} .
\end{aligned}
$$

Proof of Proposition 5. We will split this proof into four main steps.

(i) Derivation of suitable error equation. We recall that the spatial discretization with RobinNitsche interface conditions has been specifically constructed to be consistent, i.e. the continuous solution satisfies the semi-discrete variational equation

$$
\left(\frac{\mathrm{d}}{\mathrm{dt}} X, X_{h}^{*}\right)_{\mathcal{E}}+M_{h}\left(X, X_{h}^{*}\right)=L\left(X_{h}^{*}\right), \quad \forall X_{h}^{*} \in \mathcal{V}_{h}
$$

Using the projection operator $P_{h}$ we rewrite this as

$$
\begin{aligned}
\rho_{\mathrm{f}}\left(\partial_{t} \underline{v}_{\mathrm{f}}, \underline{v}_{\mathrm{f}, h}^{*}\right)_{\bar{\phi}, \Omega}+\rho_{\mathrm{s}}\left(\partial_{t} \underline{v}_{\mathrm{s}}, \underline{v}_{\mathrm{s}, h}^{*}\right)_{1-\bar{\phi}, \Omega}+\left(\partial_{t} \underline{u}_{\mathrm{s}}, \underline{u}_{\mathrm{s}, h}^{*}\right)_{\mathrm{skel}}+\frac{1}{\kappa_{\mathrm{s}}}\left(\partial_{t} p, p_{h}^{*}\right)_{(1-\bar{\phi})^{2}, \Omega} \\
+M_{h}\left(\left(P_{h}^{\mathrm{f}} X, P_{h}^{\mathrm{s}} X, P_{h}^{\mathrm{u}} X, P_{h}^{\mathrm{p}} X\right),\left(\underline{v}_{\mathrm{v}, h}^{*}, \underline{v}_{\mathrm{s}, h}^{*}, \underline{u}_{\mathrm{s}, h}^{*}, p_{h}^{*}\right)\right)=L\left(X_{h}^{*}\right) .
\end{aligned}
$$

Averaging the evaluations of this equation at times $t^{n}$ and $t^{n+1}$, and gathering consistency errors in the RHS, we obtain

$$
\begin{aligned}
& \rho_{\mathrm{f}}\left(\left(D_{\tau} P_{h}^{\mathrm{f}} X\right)^{n}, \underline{v}_{\mathrm{f}, h}^{*}\right)_{\bar{\phi}, \Omega}+\rho_{\mathrm{s}}\left(\left(D_{\tau} P_{h}^{\mathrm{s}} X\right)^{n+\frac{1}{2}}, \underline{v}_{\mathrm{s}, h}^{*}\right)_{1-\bar{\phi}, \Omega} \\
& \quad+\left(\left(D_{\tau} P_{h}^{\mathrm{u}} X\right)^{n+\frac{1}{2}}, \underline{u}_{\mathrm{s}, h}^{*}\right)_{\mathrm{skel}}+\frac{1}{\kappa_{\mathrm{s}}}\left(\left(D_{\tau} P_{h}^{\mathrm{p}} X\right)^{n}, p_{h}^{*}\right)_{(1-\bar{\phi})^{2}, \Omega} \\
&+M_{h}\left(\left(\left(P_{h}^{\mathrm{u}} X\right)^{n+\frac{1}{2}},\left(P_{h}^{\mathrm{s}} X\right)^{n+\frac{1}{2}},\left(P_{h}^{\mathrm{f}} X\right)^{n+\frac{1}{2}},\left(P_{h}^{\mathrm{p}} X\right)^{n+\frac{1}{2}}\right),\right.\left.\left(\underline{u}_{\mathrm{s}, h}^{*} \underline{v}_{\mathrm{s}, h}^{*}, \underline{v}_{\mathrm{f}, h}^{*}, p_{h}^{*}\right)\right) \\
&=\mathcal{A}^{n}\left(X_{h}^{*}\right)+L^{n+\frac{1}{2}}\left(X_{h}^{*}\right),
\end{aligned}
$$

where we denote, for any function $g$ continuous in time,

$$
\left(D_{\tau} g\right)^{n+\frac{1}{2}}=\frac{g\left(t^{n+1}\right)-g\left(t^{n}\right)}{\Delta t},\left(D_{\tau} g\right)^{n}=\frac{g\left(t^{n+1}\right)-g\left(t^{n-1}\right)}{2 \Delta t}, \quad \text { and }(g)^{n+\frac{1}{2}}=\frac{g\left(t^{n+1}\right)+g\left(t^{n}\right)}{2},
$$

with also

$$
\begin{aligned}
\mathcal{A}^{n}\left(X_{h}^{*}\right)= & \rho_{\mathrm{f}}\left(\left(D_{\tau} P_{h}^{\mathrm{f}} X\right)^{n}-\left(\partial_{t} \underline{v}_{\mathrm{f}}\right)^{n+\frac{1}{2}}, \underline{v}_{\mathrm{f}, h}^{*}\right)_{\bar{\phi}, \Omega}+\rho_{\mathrm{S}}\left(\left(D_{\tau} P_{h}^{\mathrm{s}} X\right)^{n+\frac{1}{2}}-\left(\partial_{t} \underline{v}_{\mathrm{s}}\right)^{n+\frac{1}{2}}, \underline{v}_{\mathrm{s}, h}^{*}\right)_{1-\bar{\phi}, \Omega} \\
& +\left(\left(D_{\tau} P_{h}^{\mathrm{u}} X\right)^{n+\frac{1}{2}}-\left(\partial_{t} \underline{u}_{\mathrm{s}}\right)^{n+\frac{1}{2}}, \underline{u}_{\mathrm{s}, h}^{*}\right)_{\mathrm{skel}}+\frac{1}{\kappa_{\mathrm{S}}}\left(\left(D_{\tau} P_{h}^{\mathrm{p}} X\right)^{n}-\left(\partial_{t} p\right)^{n+\frac{1}{2}}, p_{h}^{*}\right)_{(1-\bar{\phi})^{2}, \Omega}
\end{aligned}
$$

that gathers the consistency error terms. Now we introduce

$$
\tilde{X}_{h}^{n}=X_{h}^{n}-\left(P_{h} X\right)^{n}, \quad \text { with } \quad\left(P_{h} X\right)^{n}=\left(P_{h}^{\mathrm{u}} X\left(t^{n}\right), P_{h}^{\mathrm{s}} X\left(t^{n}\right),\left(P_{h}^{\mathrm{f}} X\right)^{n-\frac{1}{2}},\left(P_{h}^{\mathrm{p}} X\right)^{n-\frac{1}{2}}\right) .
$$


Subtracting (50) from (28), we obtain

$$
\begin{aligned}
\rho_{\mathrm{f}}\left(D_{\tau} \underline{\tilde{v}}_{\mathrm{f}, h}^{n+\frac{1}{2}}, \underline{v}_{\mathrm{f}, h}^{*}\right)_{\bar{\phi}, \Omega} & +\rho_{\mathrm{s}}\left(D_{\tau} \underline{\underline{v}}_{\mathrm{s}, h}^{n+\frac{1}{2}}, \underline{v}_{\mathrm{s}, h}^{*}\right)_{1-\bar{\phi}, \Omega}+\left(D_{\tau} \underline{\underline{u}}_{\mathrm{s}, h}^{n+\frac{1}{2}}, \underline{u}_{\mathrm{s}, h}^{*}\right)_{\mathrm{skel}}+\frac{1}{\kappa_{\mathrm{s}}}\left(D_{\tau} \tilde{p}_{h}^{n+\frac{1}{2}}, p_{h}^{*}\right)_{(1-\bar{\phi})^{2}, \Omega} \\
& +M_{h}\left(\left(\underline{\tilde{u}}_{\mathrm{s}, h}^{n+\frac{1}{2}}, \underline{\underline{v}}_{\mathrm{s}, h}^{n+\frac{1}{2}}, \underline{v}_{\mathrm{f}, h}^{n+1}, \tilde{p}_{h}^{n+1}\right),\left(\underline{u}_{\mathrm{s}, h}^{*}, \underline{v}_{\mathrm{s}, h}^{*}, \underline{v}_{\mathrm{f}, h}^{*}, p_{h}^{*}\right)\right)=-\mathcal{A}^{n}\left(X_{h}^{*}\right) .
\end{aligned}
$$

(ii) Error analysis by energy stability. Following the steps of the energy stability analysis of Section 3.1, we evaluate this equation with the following admissible test functions

$$
\underline{u}_{\mathrm{s}, h}^{*}=\underline{\tilde{u}}_{\mathrm{s}, h}^{n+\frac{1}{2}}, \quad \underline{v}_{\mathrm{s}, h}^{*}=\underline{\tilde{v}}_{\mathrm{s}, h}^{n+\frac{1}{2}}, \quad \underline{v}_{\mathrm{f}, h}^{*}=\underline{\tilde{v}}_{\mathrm{f}, h}^{n+1}, \quad p_{h}^{*}=\tilde{p}_{h}^{n+1} .
$$

We introduce for the evaluation of the RHS of (51)

$$
\mathcal{A}^{n}=\mathcal{A}^{n}\left(\underline{\tilde{u}}_{\mathrm{s}, h}^{n+\frac{1}{2}}, \underline{\tilde{v}}_{\mathrm{s}, h}^{n+\frac{1}{2}}, \underline{\tilde{v}}_{\mathrm{f}, h}^{n+1}, \tilde{p}_{h}^{n+1}\right),
$$

and the energy of the error at iteration $n$

$$
\tilde{\mathcal{E}}_{h}^{n}=\frac{\rho_{\mathrm{f}}}{2}\left\|\underline{\tilde{v}}_{\mathrm{f}, h}^{n}\right\|_{\bar{\phi}, \Omega}^{2}+\frac{\rho_{s}}{2}\left\|\underline{\tilde{v}}_{\mathrm{s}, h}^{n}\right\|_{1-\bar{\phi}, \Omega}^{2}+\frac{1}{2}\left\|\underline{\tilde{u}}_{\mathrm{s}, h}^{n}\right\|_{\mathrm{skel}}^{2}+\frac{1}{2 \kappa_{\mathrm{s}}}\left\|\tilde{p}_{h}^{n}\right\|_{(1-\bar{\phi})^{2}, \Omega}^{2} .
$$

Similarly to (30) we now obtain

$$
\begin{aligned}
\frac{\tilde{\mathcal{E}}_{h}^{n+1}-\tilde{\mathcal{E}}_{h}^{n}}{\Delta t}+\frac{\rho_{\mathrm{f}}}{2 \Delta t}\left\|\underline{\tilde{v}}_{\mathrm{f}, h}^{n+1}-\underline{\tilde{v}}_{\mathrm{f}, h}^{n}\right\|_{\bar{\phi}, \Omega}^{2}+\frac{1}{2 \kappa_{\mathrm{s}} \Delta t}\left\|\tilde{p}_{h}^{n+1}-\tilde{p}_{h}^{n}\right\|_{(1-\bar{\phi})^{2}, \Omega}^{2} \\
\left.+2 \| \underline{\underline{\varepsilon}}_{\underline{\mathrm{s}}, h}^{n+\frac{1}{2}}\right)\left\|_{\mu_{\mathrm{s}}, \Omega}^{2}+\right\| \underline{\tilde{v}}_{\mathrm{f}, h}^{n+1}-\underline{\underline{v}}_{\mathrm{s}, h}^{n+\frac{1}{2}} \|_{\bar{\phi}^{2} \underline{\underline{k}}_{\mathrm{f}}^{-1}, \Omega}^{2} \leq-\mathcal{A}^{n},
\end{aligned}
$$

hence, we simply have

$$
\frac{\tilde{\mathcal{E}}_{h}^{n+1}-\tilde{\mathcal{E}}_{h}^{n}}{\Delta t} \leq-\mathcal{A}^{n}
$$

(iii) Consistency errors. Now we need to control $\mathcal{A}^{n}$, which we decompose as

$$
\left\{\begin{array}{l}
\mathcal{A}_{\mathrm{f}}^{n}=\rho_{\mathrm{f}}\left(\left(D_{\tau} P_{h}^{\mathrm{f}} X\right)^{n}-\left(\partial_{t} \underline{v}_{\mathrm{f}}\right)^{n+\frac{1}{2}}, \underline{\tilde{v}}_{\mathrm{f}, h}^{n+1}\right)_{\bar{\phi}, \Omega}, \\
\mathcal{A}_{\mathrm{s}}^{n}=\rho_{\mathrm{s}}\left(\left(D_{\tau} P_{h}^{\mathrm{s}} X\right)^{n+\frac{1}{2}}-\left(\partial_{t} \underline{\mathrm{v}}_{\mathrm{s}}\right)^{n+\frac{1}{2}}, \underline{\tilde{v}}_{\mathrm{s}, h}^{n+\frac{1}{2}}\right)_{1-\bar{\phi}, \Omega} \\
\mathcal{A}_{\mathrm{u}}^{n}=\left(\left(D_{\tau} P_{h}^{\mathrm{u}} X\right)^{n+\frac{1}{2}}-\left(\partial_{t} \underline{u}_{\mathrm{s}}\right)^{n+\frac{1}{2}}, \underline{\tilde{u}}_{\mathrm{s}, h}^{n+\frac{1}{2}}\right)_{\mathrm{skel}}, \\
\mathcal{A}_{\mathrm{p}}^{n}=\frac{1}{\kappa_{\mathrm{s}}}\left(\left(D_{\tau} P_{h}^{\mathrm{p}} X\right)^{n}-\left(\partial_{t} p\right)^{n+\frac{1}{2}}, \tilde{p}_{h}^{n+1}\right)_{(1-\bar{\phi})^{2}, \Omega}
\end{array}\right.
$$

Starting with $\mathcal{A}_{\mathrm{f}}^{n}$, the linearity of $P_{h}$ gives

$$
\left(D_{\tau} P_{h}^{\mathrm{f}} X\right)^{n}-\left(\partial_{t} \underline{\mathrm{v}}_{\mathrm{f}}\right)^{n+\frac{1}{2}}=P_{h}^{\mathrm{f}}\left(D_{\tau} X\right)^{n}-\left(D_{\tau} \underline{\mathrm{v}}_{\mathrm{f}}\right)^{n}+\left(D_{\tau} \underline{\mathrm{v}}_{\mathrm{f}}\right)^{n}-\partial_{t} \underline{\mathrm{v}}_{\mathrm{f}}\left(t^{n}\right)+\partial_{t} \underline{\mathrm{v}}_{\mathrm{f}}\left(t^{n}\right)-\left(\partial_{t} \underline{\mathrm{v}}_{\mathrm{f}}\right)^{n+\frac{1}{2}} .
$$

The finite difference $\left(D_{\tau} X\right)^{n}$ satisfies the assumptions of Lemma 6 like all $X^{n}$, and therefore

$$
\begin{aligned}
\left\|P_{h}^{\mathrm{f}}\left(D_{\tau} X\right)^{n}-\left(D_{\tau} \underline{v}_{\mathrm{f}}\right)^{n}\right\|_{\bar{\phi}, \Omega} & \leq C\left\|P_{h}^{\mathrm{f}}\left(D_{\tau} X\right)^{n}-\left(D_{\tau} X\right)^{n}\right\|_{\mathcal{E}} \leq C h\left\|\left(D_{\tau} X\right)^{n}\right\|_{\mathcal{X}^{+}} \\
& \leq C h\left\|\partial_{t} X\right\|_{L^{\infty}[0, T, \mathcal{X}+]} .
\end{aligned}
$$


Then, thanks to Taylor-Lagrange inequalities,

$$
\left\|\left(D_{\tau} \underline{v}_{\mathrm{f}}\right)^{n}-\partial_{t} \underline{v}_{\mathrm{f}}\left(t^{n}\right)\right\|_{\bar{\phi}, \Omega} \leq C\left\|\partial_{t}^{2} X\right\|_{L^{\infty}[0, T, \mathcal{E}]} \Delta t,
$$

and

$$
\left\|\partial_{t} \underline{v}_{\mathrm{f}}\left(t^{n}\right)-\left(\partial_{t} \underline{\mathrm{v}}_{\mathrm{f}}\right)^{n+\frac{1}{2}}\right\|_{\bar{\phi}, \Omega} \leq C\left\|\partial_{t}^{2} X\right\|_{L^{\infty}[0, T, \mathcal{E}]} \Delta t .
$$

Therefore, the Cauchy-Schwarz inequality applied on $\mathcal{A}_{\mathrm{f}}^{n}$ gives

$$
\left|\mathcal{A}_{\mathrm{f}}^{n}\right| \leq C(h+\Delta t)\left\|\underline{\tilde{v}}_{\mathrm{f}, h}^{n+1}\right\|_{\bar{\phi}, \Omega} .
$$

Likewise, for the other terms we find,

$$
\begin{aligned}
& \left|\mathcal{A}_{\mathrm{s}}^{n}\right| \leq C(h+\Delta t)\left\|\underline{\tilde{v}}_{\mathrm{s}, h}^{n+\frac{1}{2}}\right\|_{1-\bar{\phi}, \Omega}, \\
& \left|\mathcal{A}_{\mathrm{u}}^{n}\right| \leq C(h+\Delta t)\left\|\tilde{u}_{\mathrm{s}, h}^{n+\frac{1}{2}}\right\|_{\text {skel }}, \\
& \left|\mathcal{A}_{\mathrm{p}}^{n}\right| \leq C(h+\Delta t)\left\|\tilde{p}_{h}^{n+1}\right\|_{(1-\bar{\phi})^{2}, \Omega},
\end{aligned}
$$

where we observe that the constants depend on $\left\|\partial_{t}^{2} X\right\|_{L^{\infty}[0, T, \mathcal{E}]}$ and $\left\|\partial_{t} X\right\|_{L^{\infty}\left[0, T, \mathcal{X}^{+}\right]}$.

(iv) Final error estimates. Using these consistency bounds with (52) we obtain

$$
\frac{\tilde{\mathcal{E}}_{h}^{n+1}-\tilde{\mathcal{E}}_{h}^{n}}{\Delta t} \leq C(h+\Delta t)\left(\left\|\underline{\tilde{v}}_{\mathrm{f}, h}^{n+1}\right\|_{\bar{\phi}, \Omega}+\left\|\underline{\tilde{v}}_{\mathrm{s}, h}^{n+\frac{1}{2}}\right\|_{1-\bar{\phi}, \Omega}+\left\|\underline{\tilde{u}}_{\mathrm{s}, h}^{n+\frac{1}{2}}\right\|_{\text {skel }}+\left\|\tilde{p}_{h}^{n+1}\right\|_{(1-\bar{\phi})^{2}, \Omega}\right) .
$$

Changing the index notation and using the triangular inequality, we infer

$$
\tilde{\mathcal{E}}_{h}^{i+1}-\tilde{\mathcal{E}}_{h}^{i} \leq C \Delta t(h+\Delta t)\left(\sqrt{\tilde{\mathcal{E}}_{h}^{i+1}}+\sqrt{\tilde{\mathcal{E}}_{h}^{i}}\right),
$$

hence,

$$
\sqrt{\tilde{\mathcal{E}}_{h}^{i+1}}-\sqrt{\tilde{\mathcal{E}}_{h}^{i}} \leq C \Delta t(h+\Delta t)
$$

Summing these inequalities over $i$, we have

$$
\sqrt{\tilde{\mathcal{E}}_{h}^{n}} \leq C n \Delta t(h+\Delta t)+\sqrt{\tilde{\mathcal{E}}_{h}^{0}} \leq C T(h+\Delta t)+\sqrt{\tilde{\mathcal{E}}_{h}^{0}} .
$$

which we rephrase as

$$
\left\|X_{h}^{n}-\left(P_{h} X\right)^{n}\right\|_{\mathcal{E}} \leq C(h+\Delta t) .
$$

Introducing

$$
(X)^{n}=\left(\underline{u}_{\mathrm{S}}\left(t^{n}\right), \underline{v}_{\mathrm{S}}\left(t^{n}\right),\left(\underline{v}_{\mathrm{f}}\right)^{n-\frac{1}{2}},(p)^{n-\frac{1}{2}}\right),
$$

we can apply the projection estimate (48) component by component to obtain

$$
\left\|\left(P_{h} X\right)^{n}-(X)^{n}\right\|_{\mathcal{E}} \leq C h\|X\|_{L^{\infty}\left[0, T, \mathcal{X}^{+}\right]} .
$$

In addition, of course,

$$
\left\|(X)^{n}-X\left(t^{n}\right)\right\|_{\mathcal{E}} \leq C \Delta t\left\|\partial_{t} X\right\|_{L^{\infty}[0, T, \mathcal{E}]} .
$$

Finally, gathering (53), (54) and (55), the triangular inequality concludes the proof.

\section{Remark 5}

The order of time convergence is only 1 because of the specific choices we made in Section 2.5 - namely, first-order backward Euler in the fluid and midpoint scheme for the solid - that aim at keeping the time scheme as close as possible to the splitting scheme proposed in [5]. Naturally, avoiding the shifting between fluid and solid discretizations - choosing for example a Crank-Nicolson method [14] for the fluid part - would provide a convergence in $\Delta t^{2}$. 


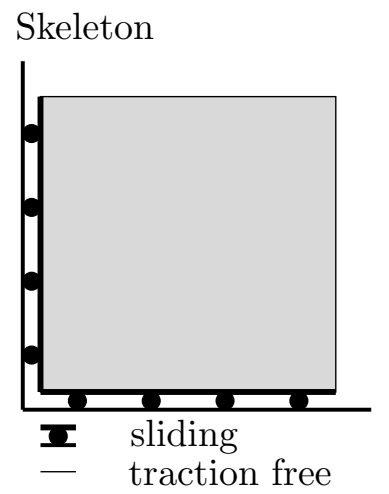

Fluid

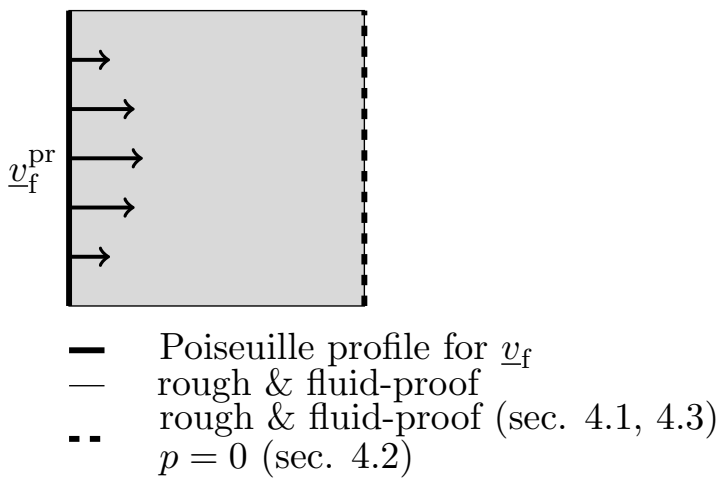

Figure 1: Swelling test boundary conditions

\section{Numerical illustrations and assessments}

In this section, we present various numerical results to illustrate the relevance of the above study and of Proposition 5 that ensures the total convergence of the numerical scheme (28) under various conditions.

Remark 6 (Solid discretization and the condition (35))

We see in the proof of Proposition 4 - in the construction of $X_{h}^{4}$, specifically - that pressure is essentially stabilized by the fluid velocity, therefore the inf-sup condition (34) plays a prominent role. By contrast, the inf-sup condition on the solid part (35) is only used to construct a convenient lifting operator. In fact, in all our numerical results presented below, we used solid displacements and velocities in the same finite element space as the pressure, i.e. $P_{1}$ here, which clearly violates (35). Nevertheless, the numerical instabilities that we observed when $\mathcal{W}_{h}^{f} \times \mathcal{W}_{h}^{s} \times \mathcal{Q}_{h}$ is $P_{1} \times P_{1} \times P_{1}$ appeared to be effectively cured with more adequate discretization choices for the fluid velocity, whereas other tests revealed that they were not circumvented by inf-sup stable discretizations on the solid side.

We consider here several situations to illustrate how numerical perturbations arise when approaching solid incompressibility - i.e. when the parameter $\kappa_{\mathrm{s}}$ grows - and the importance of satisfying the inf-sup conditions to circumvent these perturbations. The inf-sup condition (34) can be difficult to satisfy in practice with finite element spaces, as it requires a non-natural variable for the discretization of the fluid velocity, i.e. $\bar{\phi} \underline{v}_{\mathrm{f}}$. Therefore, it is natural to attempt to relax (34) into (40), and to investigate to what extent this simplification may affect the numerical stability when $\bar{\phi}$ is not homogeneous.

In Section 4.1, we illustrate the importance of $(34)$ when $\bar{\phi}$ is spatially homogeneous. In Section 4.2 , we study the effect of spatial variations of $\bar{\phi}$ on spatial convergence of the scheme, for different discretization methods, and perform a numerical study of the relevance of (34) compared to (40). In Section 4.3, we extend our study to check that our conclusions remain relevant in the non-linear framework $[5,6]$.

In all cases, we consider a 2D swelling test problem as proposed in [5] with a prescribed fluid velocity on one side of the domain, see Figure 1 . The numerical values of the parameters are given in Table 1, with the relationships $\lambda=\kappa-\frac{4\left(\kappa_{1}+\kappa_{2}\right)}{3}$ and $\mu=2\left(\kappa_{1}+\kappa_{2}\right)$ for the Lamé coefficients used in the Saint-Venant-Kirchhoff hyperelastic potential (11). Solid viscosity is set to zero. 


\begin{tabular}{l||l|l|l|l|l|l|l|l|l|l} 
Parameter & $|\Omega|$ & $\Delta t$ & $\gamma$ & $\kappa, \kappa_{1}$ & $\kappa_{2}$ & $\rho_{\mathrm{s}}, \rho_{\mathrm{f}}$ & $\mu_{\mathrm{f}}$ & $\bar{\phi}$ & $\underline{\underline{D}}_{\mathrm{f}}$ & $\max \left(\underline{v}_{\mathrm{f}}^{\mathrm{pr}}\right)$ \\
\hline Section 4.1 & $10^{-4}$ & $10^{-3}$ & $210^{4}, 210^{5}$ & $210^{3}$ & 33 & $10^{3}$ & 0.035 & 0.1 & $10^{7} \underline{\underline{I}}$ & $10^{-4}$ \\
Section 4.2 & $10^{-4}$ & $10^{-3}$ & $210^{4}, 210^{6}$ & $210^{3}$ & 33 & $10^{3}$ & 0.035 & $\bar{\phi}^{\mathrm{nc}}$ & $10^{7} \overline{\bar{I}}$ & $10^{-3}$ \\
Section 4.3 & $10^{-4}$ & $10^{-3}$ & 200 & $210^{3}$ & 33 & $10^{3}$ & 0.035 & 0.1 & $10^{7} \underline{\underline{I}}$ & 0.05
\end{tabular}

Table 1: Parameter values (all units SI)

\subsection{Inf-sup compatible finite element spaces for fluid velocity and pressure}

First, we assume that $\bar{\phi}$ is homogeneous in space. Therefore, the inf-sup condition (34) reduces to the classical condition (40), widely studied in the context of the discretization of Stokes problems. It is well known that (40) is satisfied for instance when $\mathcal{W}_{h}^{f} \times \mathcal{Q}_{h}$ is in $P_{1}^{b} \times P_{1}$, but is violated for $P_{1} \times P_{1}$.

The spatial step-length $h$ will vary from $1 / 800$ to $1 / 6400$ and a numerical reference solution is obtained with $h=1 / 7200$. Five quadrature points are used, and $\kappa_{\mathrm{s}}$ will take the values $2 e 2$, $2 e 4$ and $2 e 8$. The test case here is as follows (see Figure 1):

- $\underline{v}_{\mathrm{s}} \cdot \underline{n}=0$ on the bottom and left faces;

- Horizontal fluid velocity is imposed with a Poiseuille profile of amplitude $10^{-4}$ on the left face;

- $\underline{v}_{\mathrm{s}}=\underline{v}_{\mathrm{f}}$ on the bottom, top and right faces.

Figure 3 illustrates the pressure instabilities that arise with the choice $P_{1} \times P_{1}$, compared with the inf-sup compatible case $P_{1}^{b} \times P_{1}$. In addition, in Figure 2 we plot numerical errors in $L^{2}$-norm for fluid velocity and pressure with respect to the numerical reference solutions. We see no sign of numerical instability - or altered convergence - when using the inf-sup compatible spaces $P_{1}^{b} \times P_{1}$. By contrast, when using $P_{1} \times P_{1}$ pressure oscillations appear for large values of $\kappa_{\mathrm{S}}$, and convergence curves are also altered, in particular for the pressure. In fact, we notice that these instabilities grow with $\gamma$. For smaller $\gamma$, they also appear, but disturb less the overall solution. However, in this case the interface condition cannot be imposed with as much precision, see Figure 4.

\subsection{Discretization for a non-spatially-homogeneous $\bar{\phi}$}

We consider the non-spatially-homogeneous $\bar{\phi}$ given by

$$
\bar{\phi}=\bar{\phi}^{\mathrm{nc}}(x, y)=0.5+0.4 \sin \left(\frac{10 \pi x}{L}\right) \sin \left(\frac{10 \pi \mathrm{y}}{L}\right),
$$

and illustrate in Figure 5 the relevance of solving the fluid mixed problem with unknowns $\left(\underline{w}_{\mathrm{f}}=\bar{\phi} \underline{v}_{\mathrm{f}}, p\right)$ in $\mathcal{W}_{h}^{f} \times \mathcal{Q}_{h}$, instead of $\left(\underline{v}_{\mathrm{f}}, p\right)$. We consider the same test as before, but with a Poiseuille amplitude of $10^{-3}$ on the left side and $p=0$ on the right side. We use here $\kappa_{\mathrm{s}}=2 e 4$ (results are similar for 2e8), 5 quadrature points, and $\gamma=2 e 4$ (results are similar for $2 e 6$ ). We compare the spatial convergence curves for the following finite element choices:

- Choice 1: according to the above study, in order to satisfy (34) the unknowns are $\left(\underline{w}_{\mathrm{f}}=\right.$ $\left.\bar{\phi} \underline{v}_{\mathrm{f}}, p\right)$ in $P_{1}^{b} \times P_{1}$. Then, we get $\underline{v}_{\mathrm{f}}$ as the $H^{1}$ projection of $\underline{w}_{\mathrm{f}} / \bar{\phi}$

- Choice 2: unknowns are $\left(\underline{v}_{\mathrm{f}}, p\right)$ in $P_{1}^{b} \times P_{1}$, and $\bar{\phi}$ is evaluated at the integration points 

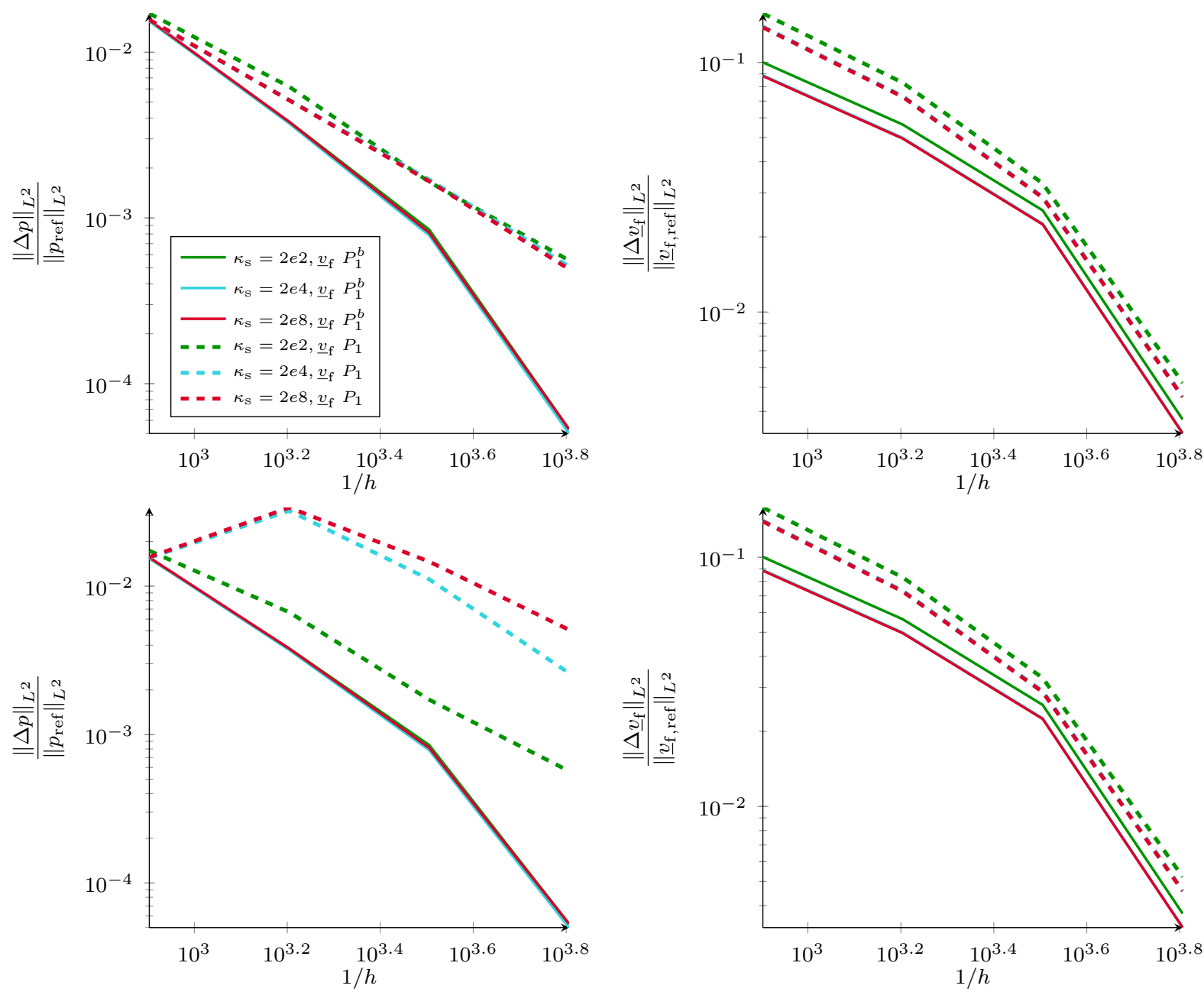

Figure 2: Relative error $L^{2}$-norms for pressure (left) and fluid velocity (right) at $\mathrm{t}=2$; top row $\gamma=2 e 4$, bottom row $\gamma=2 e 5$ 

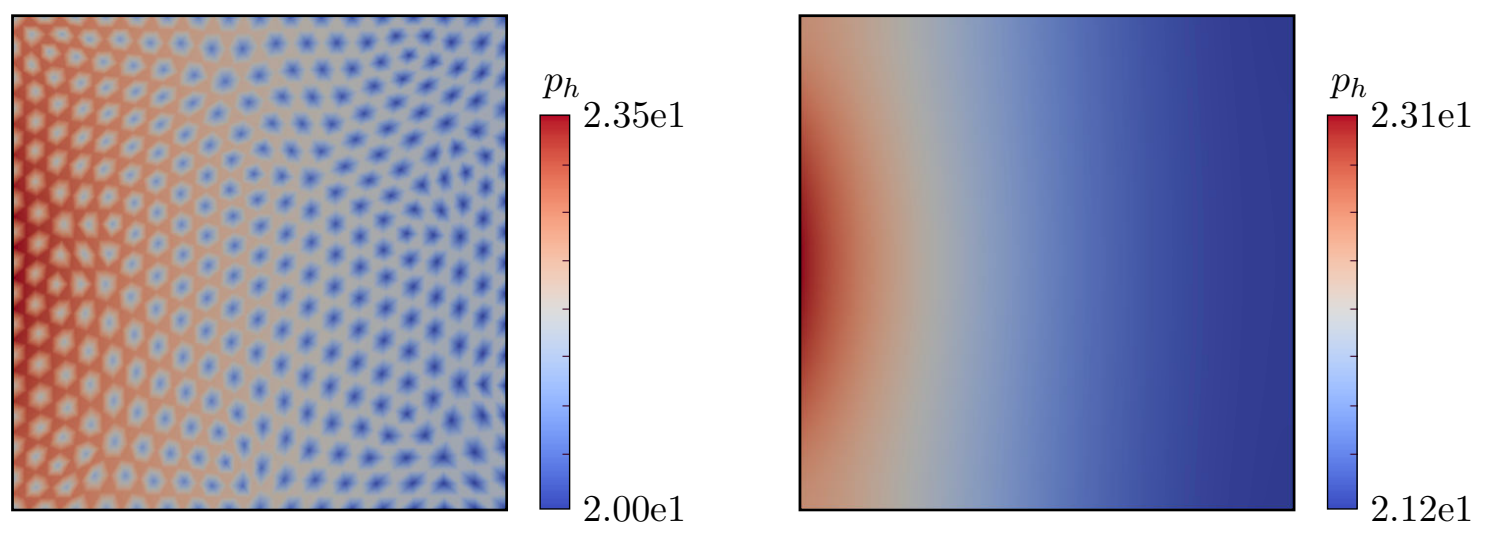

Figure 3: Pressure profile for $\underline{v}_{\mathrm{f}} P_{1}$ (left) vs. $P_{1}^{b}$ (right); $1 / h=3200, \kappa_{\mathrm{s}}=2 e 8, \gamma=2 e 5$

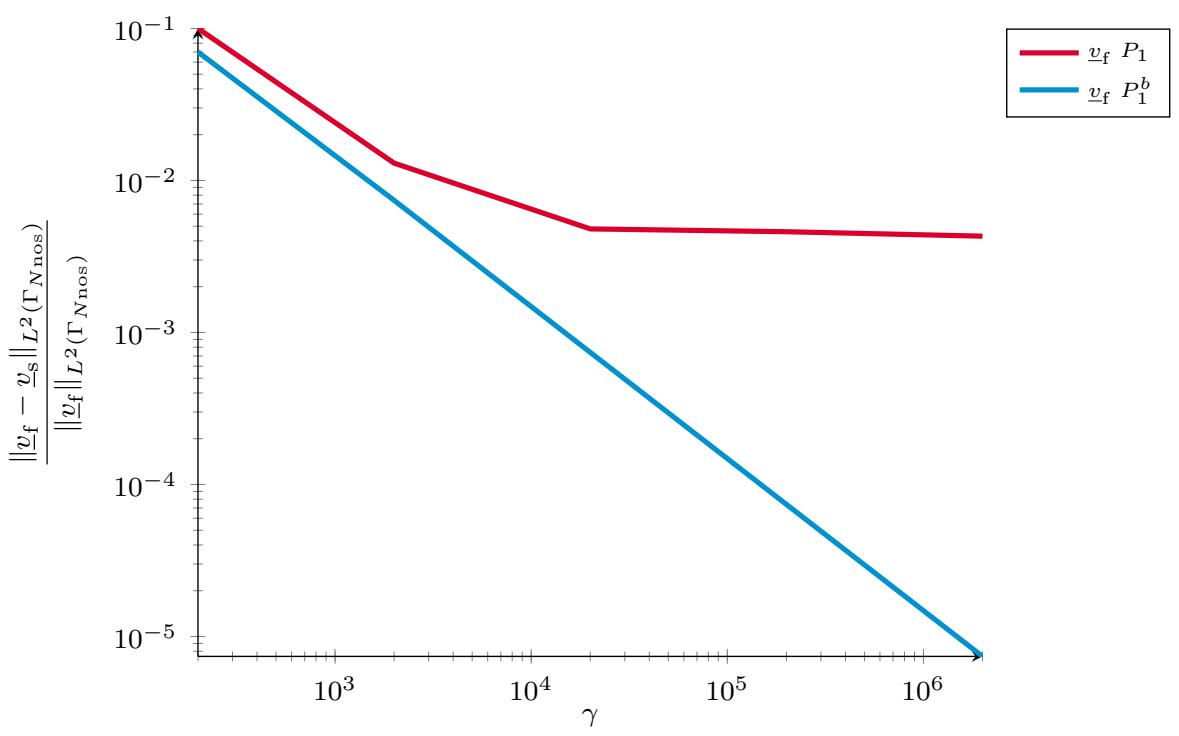

Figure 4: Error in interface condition when $\gamma$ varies

- Choice 3: unknowns are $\left(\underline{v}_{\mathrm{f}}, p\right)$ in $P_{1}^{b} \times P_{1}$, and $\bar{\phi}$ is interpolated into $P_{1}$.

In the second and third methods, (34) is violated but (40) is satisfied. We see in Figure 5 that even if (34) gives better convergence for fluid pressure and velocities, satisfying the relaxed conditions (40) gives reasonably good numerical results. This is interesting as (34) is in most cases - especially in a nonlinear framework - much harder to impose at the discrete level than (40), as it requires to choose $\underline{w}=\phi \underline{v}_{\mathrm{f}}$ as discrete unknown for the fluid velocity.

\subsection{Extension to non-linear framework}

In this last scenario, we illustrate the relevance of this study for choosing adequate spatial discretization in a nonlinear framework, in which case the fluid fraction $\phi$ is an unknown in itself, hence, will vary in time and space. We perform simulations for the test case of Section 4.1, using the splitting time scheme proposed in [5] in the Arbitrary Lagrangian-Eulerian formalism. We consider a Saint-Venant-Kirchhoff hyperelastic potential for the solid, with $\kappa_{\mathrm{s}}=2 e 5$, $1 / h=1600$, and other parameters given in Table 1 . We compare the pressure obtained when discretizing $\underline{v}_{\mathrm{f}}$ in $P_{1}$ and in $P_{1}^{b}$. 

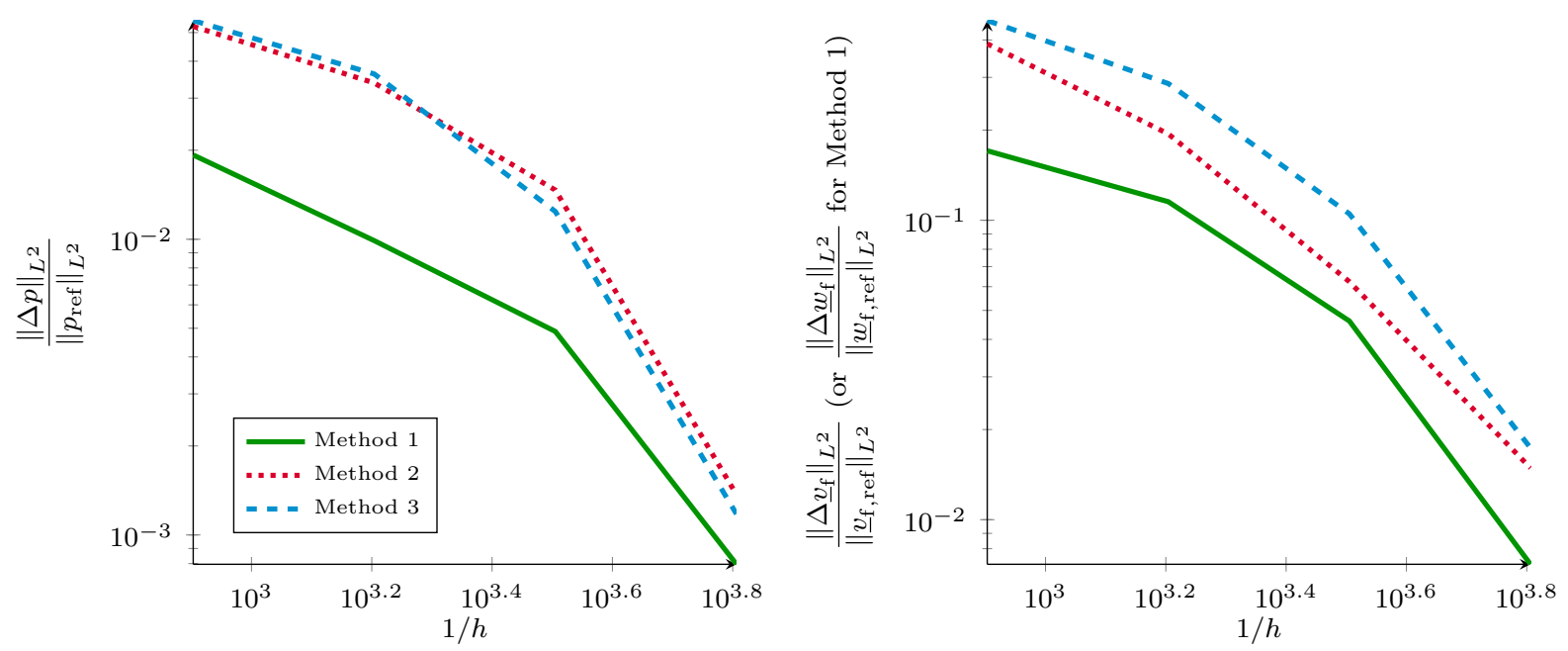

Figure 5: Relative error $L^{2}$-norms for pressure (left) and fluid velocity (right) at $\mathrm{t}=2$ for several discretization choices when $\bar{\phi}$ is non-homogeneous
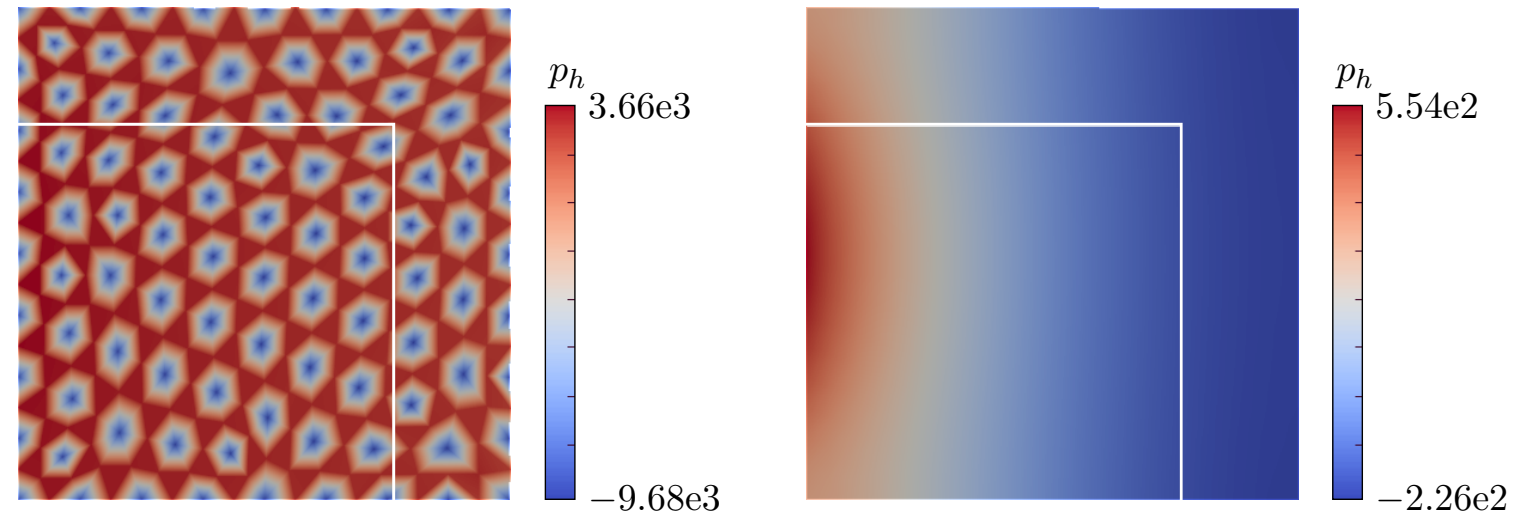

Figure 6: Pressure profile for $\underline{v}_{\mathrm{f}} P_{1}$ (left) vs. $P_{1}^{b}$ (right); nonlinear case

In order to satisfy (34), our discrete variables in the implicit fluid projection substep should be $\left(\phi \underline{v}_{\mathrm{f}}, p, m\right)$. However, the $H^{1}$ projection that enables us to go back from $\left(\phi \underline{v}_{\mathrm{f}}\right)$ to $\underline{v}_{\mathrm{f}}$ (necessary for the explicit step) needs to reach $\phi$ at the integration points. The fluid fraction $\phi$ varies in time and space, and its definition calls functions that are defined on different meshes. Simulations are performed with FreeFem ++ [13] that present many advantages, but does not allow such manipulations. In the light of the relatively good results of the third method of Section 4.2, it is legitimate to relax (34) into (40), which is much easier to verify in the implementation. We illustrate in Figure 6 that the condition (40) is sufficient to prevent pressure oscillations, indeed.

\section{Conclusion}

We have derived a linearized formulation problem (8) of the general poromechanical formulation (5) previously proposed in [6]. We have then proposed a complete discretization strategy - in space and time - for this linearized formulation and obtained an error estimate for the discrete solution. Moreover, spatial discretization was performed with a special concern for incompressibility issues - that may lead to pressure oscillations and numerical locking, in par- 
ticular - and the final error estimate is uniform with respect to solid compressibility, indeed. In order to achieve this, the finite element spaces considered are assumed to satisfy various assumptions, and in particular two specific inf-sup conditions, see Proposition 5.

In practice, as discussed in Section 4, satisfying these specific inf-sup conditions can be very cumbersome. Nevertheless, our numerical tests indicate that only one inf-sup condition is essential, i.e. (34) pertaining to the combined choice of fluid velocity and pressure finite element spaces. Moreover, relaxing this non-standard inf-sup condition into the usual Stokes-associated condition (40) is a possible choice - albeit suboptimal from the accuracy point of view - as observed in the numerical assessments.

We point out that the numerical finding that only one inf-sup condition may be needed to ensure convergence is perfectly compatible with the fact that only one constraint arises in the solid incompressible limit, i.e. (17). Accordingly, a natural perspective for this work would be to improve the analysis to totally dispense with the second condition.

Acknowledgement: The authors are very grateful to Céline Grandmont (Inria) for some insightful discussions on existence results and techniques of proof for fluid-structure interaction problems.

\section{References}

[1] A. Bensoussan, M.C. Delfour, G. Da Prato, and S.K. Mitter. Representation and Control of Infinite Dimensional Systems. Birkhauser Verlag, second edition edition, 2007.

[2] F. Brezzi and M. Fortin. Mixed and Hybrid Finite Element Methods. Springer, 1991.

[3] E. Burman and M.A. Fernández. Stabilized explicit coupling for fluid-structure interaction using Nitsche's method. C.R. Math., 345(8):467-472, 2007.

[4] E. Burman and M.A. Fernández. Stabilization of explicit coupling in fluid-structure interaction involving fluid incompressibility. Comput. Methods Appl. Mech. Eng., 198(5):766 $784,2009$.

[5] B. Burtschell, D. Chapelle, and P. Moireau. Effective and energy-preserving time discretization for a general nonlinear poromechanical formulation. Comput. Struct., 182(x):313-324, 2017.

[6] D. Chapelle and P. Moireau. General coupling of porous flows and hyperelastic formulations-From thermodynamics principles to energy balance and compatible time schemes. Eur. J. Mech. B. Fluids, 46:82-96, 2014.

[7] P.G. Ciarlet. Mathematical Elasticity - Volume I: Three-Dimensional Elasticity. NorthHolland, 1988.

[8] P. Clément. Approximation by finite element functions using local regularization. Rev. Fr. Automat. Infor., 9(2):77-84, 1975.

[9] F. Costanzo and S.T. Miller. An arbitrary Lagrangian-Eulerian finite element formulation for a poroelasticity problem stemming from mixture theory. Comput. Methods Appl. Mech. Eng., 323:64-97, 2017.

[10] O. Coussy. Poromechanics. John Wiley and Sons, 2004. 
[11] M.A. Fernández, J.F. Gerbeau, and C. Grandmont. A projection semi-implicit scheme for the coupling of an elastic structure with an incompressible fluid. Int. J. Numer. Methods Eng., 69(4):794-821, January 2007.

[12] J.F. Gerbeau and M. Vidrascu. A Quasi-Newton Algorithm Based on a Reduced Model for Fluid-Structure Interaction Problems in Blood Flows. ESAIM: M2AN, 37(4):631-647, November 2003.

[13] F. Hecht. New development in FreeFem++. J. Numer. Math., 20(3-4):251-265, 2012.

[14] P. Le Tallec and P. Hauret. Energy conservation in fluid structure interactions. In O. Pironneau Y. Kuznetsov, P. Neittanmaki, editor, Numerical Methods for Scientific Computing / Variational Problems and Applications - CIMNE Barcelona, 2003.

[15] J.L. Lions and E. Magenes. Non-Homogeneous Boundary Value Problems and Applications, volume 1. Springer-Verlag, 1972.

[16] J. Nitsche. Über ein Variationsprinzip zur Lösung von Dirichlet-Problemen bei Verwendung von Teilräumen, die keinen Randbedingungen unterworfen sind. Abh. Math. Sem. Univ. Hamburg, 36:9-15, 1971.

[17] A.T. Vuong, C. Ager, and W.A. Wall. Two finite element approaches for Darcy and Darcy-Brinkman flow through deformable porous media - Mixed method vs. NURBS based (isogeometric) continuity. Comput. Methods Appl. Mech. Eng., 305:634-657, June 2016. 